\title{
Random quantum batteries
}

\author{
Francesco Caravelli $\odot,{ }^{1,2}$ Ghislaine Coulter-De Wit, ${ }^{2}$ Luis Pedro García-Pintos, ${ }^{2,3,4}$ and Alioscia Hamma ${ }^{2,5}$ \\ ${ }^{1}$ Theoretical Division and Center for Nonlinear Studies, Los Alamos National Laboratory, Los Alamos, New Mexico 87545, USA \\ ${ }^{2}$ Department of Physics, University of Massachusetts, Boston, Massachusetts 02125, USA \\ ${ }^{3}$ Joint Center for Quantum Information and Computer Science, NIST/University of Maryland, College Park, \\ Maryland 20742, USA \\ ${ }^{4}$ Joint Quantum Institute, NIST/University of Maryland, College Park, Maryland 20742, USA \\ ${ }^{5}$ Université Grenoble Alpes, CNRS, LPMMC, 38000 Grenoble, France
}

(Received 18 October 2019; accepted 6 March 2020; published 28 April 2020)

\begin{abstract}
Quantum nanodevices are fundamental systems in quantum thermodynamics that have been the subject of profound interest in recent years. Among these, quantum batteries play a very important role. In this paper we lay down a theory of random quantum batteries and provide a systematic way of computing the average work and work fluctuations in such devices by investigating their typical behavior. We show that the performance of random quantum batteries exhibits typicality and depends only on the spectral properties of the time evolving operator, the initial state, and the measuring Hamiltonian. At given revival times a random quantum battery features a quantum advantage over classical random batteries. Our method is particularly apt to be used both for exactly solvable models like the Jaynes-Cummings model or in perturbation theory, e.g., systems subject to harmonic perturbations. We also study the setting of quantum adiabatic random batteries.
\end{abstract}

DOI: 10.1103/PhysRevResearch.2.023095

\section{INTRODUCTION}

Quantum batteries [1-8] are a fundamental concept in quantum thermodynamics [9-17], and they have attracted interest as part of research in nanodevices that can operate at the quantum level [18-20]. Tools and insights from quantum information theory have provided a natural bedrock for the description of quantum nanodevices and quantum batteries from the point of view of resource and information theory [7,21-29].

In a closed quantum system, a battery can be modeled by a time-dependent Hamiltonian $H(t)$ evolving from an initial $H_{0}$ to a final $H_{1}$. The system is initialized in a state $\rho$ and, given that the entropy of the battery is constant under unitary evolution, the work extracted is given by the difference between the initial and final energies as measured in $H_{0}$ [2].

In this paper, we lay down the theory of random quantum batteries (RQB). The randomness lies in the initial state $\rho$, the Hamitonian defining the units of the energy $H_{0}$, and the time-evolution operator $U_{t}$. We are concerned with the average work extractable by (or storable in) such a device and its fluctuations.

The main results of this paper are as follows: (i) proving a typicality result for the extracted work in a large class of timedependent quantum systems. We show that-as the dimension $n$ of the Hilbert space becomes large-the extracted work

Published by the American Physical Society under the terms of the Creative Commons Attribution 4.0 International license. Further distribution of this work must maintain attribution to the author(s) and the published article's title, journal citation, and DOI. is almost always given by the difference in energy between the initial state and the completely mixed state, amplified by a quantum efficiency factor $1+Q / n^{2}$ that depends solely on the distribution of the eigenvalues of the exponential of the time-dependent perturbation operator $K$. For $Q=0$, this result can be obtained by a classical system at infinite temperature. A random quantum battery can do it with limited energy resources. A nonvanishing $Q$ is a contribution that is purely quantum and depends on the constructive interference between different eigenvalues of $K$. The second main result is (ii) to provide a general method to study the average extractable work and its fluctuations in perturbation theory, which is essential to obtain results for physically relevant systems beside few exactly solvable models. We study as an example the Jaynes-Cummings model with a harmonic perturbation. Finally (iii), we study the case of adiabatic random quantum batteries, that is, batteries that operate slowly, so that there is no inversion of the populations of the energy levels. We show that also adiabatic random quantum batteries feature typicality in the large Hilbert space dimension $n$ limit.

There is a large interest in typical properties in batteries due to the effect of disorder and the environment. In Ref. [30] a model of quantum battery based on a spin chain is studied where randomness is introduced as disorder in the couplings of the Hamiltonian $H_{0}$. In Ref. [31] the disorder is introduced in the interaction Hamiltonian which is chosen to be in the many-body localized phase. In Refs. [32,33], the work statistics in the scenario of a random quantum quench are computed, and it is shown that the knowledge of the work statistics in this setting yields information on the Loschmidt echo dynamics. The importance of work fluctuations in quantum thermodynamics in a different setting than ours was also studied in Ref. [34]. 


\section{SETUP}

In this section, we are studying the typical behavior of random batteries when the interaction Hamiltonian is a random operator. The importance of this approach lies in the fact that typicality is a powerful argument to establish general features in quantum thermodynamics. As an example, typicality of entanglement in Hilbert space can be used to explain thermalization in a closed quantum system [35]. On the other hand, this approach is useful to argue about the robustness of a model of quantum battery.

We model the quantum battery in the following way. Start with a finite-dimensional Hilbert space $\mathcal{H}=\mathbb{C}^{n}$ and timedependent Hamiltonians $H(t) \in \mathcal{B}(\mathcal{H})$, that is, a bounded Hermitian operator on $\mathcal{H}$. The initial state of the system will be denoted by $\rho$ and its time evolution by $\rho_{t}=\mathcal{U}_{t} \rho \equiv$ $U_{t} \rho U_{t}^{\dagger}$, where the unitary evolution operator is given by the time-ordered product $U_{t}=\mathcal{T} \exp \left[-i \int_{0}^{t} H(s) d s\right]$. We model the Hamiltonian in two ways. In the first scenario we consider the time dependence as a perturbation of a time-independent Hamiltonian $H_{0}$, that is, $H_{G}(t)=H_{0}+V_{G}(t)$. The subscript $G$ indicates the randomness of the perturbation which we take to be $V_{G}(t)=G^{\dagger} V(t) G$, where $G$ is a unitary representation of the unitary group on $\mathbb{C}^{n}$. In the second scenario we consider the time evolution generated by adiabatic evolution induced by a Hamiltonian $H_{G}(t)$, where the $G_{t}$ is a family of unitary operators that rotates the projectors onto the subspaces of a given energy. The discussion of the adiabatic scenario is deferred to Sec. IV C.

In both settings, we can similarly model randomness in the initial state $\rho$ or Hamiltonian $H_{0}$ also by random rotations $\rho_{G}=G \rho G^{\dagger}$ and $H_{G}=G^{\dagger} H_{0} G$. Loosely speaking, we will refer to the spectra of the initial state, of the measuring Hamiltonian $H_{0}$, and of the evolution operator

$$
K=\mathcal{T} \exp \left[-i \int_{0}^{t} V(s) d s\right]
$$

collectively as the battery spectrum. Notice that all these randomizations preserve the battery spectrum. This is a crucial point in this paper, as we are interested in ensembles of quantum batteries with a given spectrum. Randomizing also over the spectrum will yield, as we shall see, trivial results.

In our setting the system is closed and evolves unitarily and the entropy of the battery does not change. Thus the work extracted from the quantum battery is given by

$$
W(t)=\operatorname{Tr}\left[\left(\rho-\rho_{t}\right) H_{0}\right]
$$

(or ergotropy [1,36]).

As mentioned before, this approach is different from the type of disorder in the couplings considered in the literature [30-33], as for us disorder is a random rotation $G$ that mantains the spectrum of the eigenvalues of the perturbation $V_{G}(t)$ (the interaction).

A simple example which clarifies how our disorder affects the extracted work $W$ is the following single spin case inspired by nuclear magnetic resonance (NMR). We consider a Hamiltonian of the form $H_{0}=\hat{\sigma}_{x}+H_{\text {int }}$, where $H_{\text {int }}=g \vec{\sigma} \cdot \vec{B}(t)$ and $\vec{B}(t)=\left(b_{x}(t), b_{y}(t), b_{z}(t)\right)$ is the external magnetic field and $\vec{\sigma}$ the Pauli matrices. The spectrum of the interaction is effectively dependent only on the norm of the external field $\vec{B}$, which can, however, be directed in all directions. We focus on an average which keeps the spectrum of the interaction constant but rotates its basis. A two-level system example is the Jaynes-Cummings model of optics, on which we focus our attention in a random electromagnetic background. Precise experiments in these systems exist and thus provide a good background for testing the typical behavior of (random) quantum batteries [37,38].

The Hamiltonian $H_{0}$ defines the energy measurement, that is, the amount of energy stored in the battery. If we had access to any possible random Hamiltonian $H(t)$, then we would expect that the average state $\rho_{t}$ after the evolution should be the completely mixed state, in which case the average work extracted would be $\langle W\rangle=E_{0}-\operatorname{Tr} H_{0} / n$. This work is positive (that is, the battery has discharged) if the initial energy was larger than the energy in the completely mixed state or it has charged if the initial state was populating the lower levels of $H_{0}$. Notice that this setting we have arbitrary Hamiltonians $H(t)$ that can access arbitrary high energies as measured by $H_{0}$. Instead, we ask how much work can be extracted if we have limited energetic resources, that is, when the spectra of $H_{0}$ and $V(t)$ are fixed. This motivates our setting in terms of rotations of the time-dependent part of the Hamiltonians as $H_{G}(t)=H_{0}+V_{G}(t)$.

In the following, we are interested in the average work obtained by averaging over initial states $\rho$, the measurement of energy Hamiltonian $H_{0}$, and the time-dependent Hamiltonian $H_{G}(t)$. The averages are performed according to the Haar measure on $\mathbb{C}^{n}$. The fluctuations of work are defined through the same Haar averaging as $\Delta W^{2}=\left\langle(W-\langle W\rangle)^{2}\right\rangle$. In the following, the symbol $\langle X\rangle$ will represent the Haar average $\langle X\rangle=\int d U G_{U}^{\dagger} X G_{U}$, where $G_{U}$ is the suitable representation of the unitary group. We use standard techniques for the Haar averaging (see, e.g., Refs. [39-41]) to compute the average and variances according to the Haar measure.

\section{A. Work and quantumness}

A quick calculation shows that $W(t)=\operatorname{Tr}\left\{U_{t}^{\dagger} H_{0}\right.$ $\left.\left[\rho, U_{t}\right]\right\}=\operatorname{Tr}\left\{\rho\left[U_{t}, U_{t}^{\dagger} H_{0}\right]\right\}=\operatorname{Tr}\left\{U_{t}\left[U_{t}^{\dagger} H_{0}, \rho\right]\right\}$. These expressions imply that the extractable work depends on the lack of commutativity between the initial state $\rho$, the evolution operator $U_{t}$, and the Hamiltonian $H_{0}$. Moreover, they show that the coherence of the initial state in the eigenbasis of the evolution operator is necessary to have nonvanishing extractable work from a quantum battery [42-50]. In particular, if the initial state is a steady state for the unitary evolution, the work is identically zero and so are work fluctuations. It is interesting that coherence in two different bases plays a role, which calls for a multibasis definition of coherence from the resource theoretic point of view. In the following, we will see that this lack of commutativity takes the form of out-of-time-order correlators (OTOC), which is a hint to the connection between performance of quantum batteries and quantum chaos [32]. Notice that these expressions are also valid in the interaction picture $U_{I}=\exp \left(i H_{0} t\right) U_{t}=$ $\mathcal{T} \exp \left[-i \int_{0}^{t} V_{G}(s) d s\right]=G \mathcal{T} \exp \left[-i \int_{0}^{t} V(s) d s\right] G^{\dagger} \equiv G K G^{\dagger}$, an expression that will become useful later. Bounds on the 
stored and extracted energy have been obtained recently in Ref. [51], also in terms of the quantum Fisher information for the power $P_{t}=\frac{d}{d t} W$.

As we remarked above, with no limit on energetic resources one can bring the system on average in the completely mixed state. A quantum channel that just dephases the system and mixes up the populations can achieve the same final result. The same result can be obtained by a classical system working at infinite temperature. Consequently, we are also interested in whether quantum coherence plays a specific role in outperforming the mixed-state case. As we shall see, partial revivals due to the build-up of quantum coherence provide a quantum advantage.

\section{AVERAGE WORK AND FLUCTUATIONS IN RQBs}

In this section, we show how the average work and its fluctuations behave in quantum random batteries when we randomize over the initial states $\rho$, the measuring Hamiltonian $H_{0}$, or the interaction $V(t)$. In all cases, this average is obtained by rotating these operators by a random unitary operator and by taking the Haar average.

Let us start by computing the average work obtained by a generic quantum evolution and averaging over all the initial states. It should not be surprising that the average extracted work amounts to zero. Indeed, we have

$$
\langle W\rangle_{\rho}=\operatorname{Tr}\left[\langle\rho\rangle\left(H_{0}-U^{\dagger} H_{0} U\right)\right]=\frac{1}{n} 11 \operatorname{Tr} \delta H_{0}=0,
$$

where we defined the traceless operator $\delta H_{0} \equiv$ $\left[H_{0}-U^{\dagger} H_{0} U\right]$ and have used that the Haar-average state in the Hilbert space is $\langle\rho\rangle=1 / n \mathbb{1}$. However, the fluctuations are not trivial [34]. Details of the calculation are in Appendix A 1. We obtain

$$
\Delta W_{\rho}^{2}=2 \frac{n \operatorname{Tr} \rho^{2}-1}{n\left(n^{2}-1\right)}\left[\operatorname{Tr} H_{0}^{2}-\operatorname{Tr}\left(H_{0} U_{t}^{\dagger} H_{0} U_{t}\right)\right] .
$$

It is remarkable that the maximum of the fluctuations are reached for a pure state, whereas they decrease with the purity of the initial state and are identically zero if the system is initialized in the completely mixed state. Similarly, fluctuations in the work are smaller the larger the fluctuations in the eigenvalues of $H_{0}$. Notice that the time-dependent part has the form of a (two-point) out-of-time-ordered correlator (OTOC) $[52,53]$.

What happens instead if we choose randomly the measuring Hamiltonian $H_{0}$ ? As we said above, we model this family of Hamiltonians as $H_{G}=G^{\dagger} H_{0} G$. This is a sensible definition as it gives us results that still depend on the spectrum of the Hamiltonian. Again, it should not surprise that the average work is zero, since

$$
\langle W\rangle_{H_{0}}=\operatorname{Tr}\left[\left(\rho-\rho_{t}\right)\left\langle H_{0}\right\rangle\right]=\frac{\operatorname{Tr} H_{0}}{n} \operatorname{Tr}\left(\rho-\rho_{t}\right)=0
$$

as the average of every operator in the trivial representation is proportional to the identity, and $\rho-\rho_{t}$ is traceless. Some tedious calculations in Appendix A 2 show that the work fluctuations are given by

$$
\Delta W_{H_{0}}^{2}=\left\langle W^{2}\right\rangle_{H_{0}}=\frac{2 n}{n^{2}-1} \Delta H_{0}^{2} \operatorname{Tr}\left(\rho^{2}-\rho \rho_{t}\right),
$$

where $\Delta H_{0}^{2}=\frac{1}{n} \operatorname{Tr} H_{0}^{2}-\frac{1}{n^{2}}\left(\operatorname{Tr} H_{0}\right)^{2}$ are the fluctuations of the eigenvalues of $H_{0}$, namely the fluctuations of $H_{0}$ in the completely mixed state. Again, the time-dependent part $\operatorname{Tr}\left(\rho \rho_{t}\right)$ has the form of an OTOC. The connection between OTO correlators and Loschmidt echo has recently been investigated in Ref. [27]. In terms of the 2-norm fidelity $\mathcal{F}_{2}(\rho, \sigma)=$ $\operatorname{Tr}(\rho \sigma) / \max \left[\operatorname{Tr} \rho^{2}, \operatorname{Tr} \sigma^{2}\right]$ and the Loschmidt echo $\mathcal{L}_{t}=$ $\mathcal{F}_{2}\left(\rho \rho_{t}\right)$, we have

$$
\Delta W_{H_{0}}^{2}=\frac{2 n}{n^{2}-1} \Delta H_{0}^{2} \operatorname{Tr} \rho^{2}\left[1-\mathcal{L}_{t}\right] .
$$

Notice that as $\mathcal{L}_{t}$ is typically scaling as $n^{-2}$ [54], the average fluctuations are determined only by the fluctuations in $H_{0}$ and the purity of the initial state. However, at specific, revival times, there is a spike in fluctuations. Moreover, if we consider the average work over a large time $T$, then the average Loschmidt echo becomes the purity of the the completely dephased state in the basis of the Hamiltonian, $\bar{\rho}$, and the above expression reads

$$
{\overline{\Delta W^{2}}}^{T}=\frac{2 n}{n^{2}-1} \Delta H_{0}^{2} \operatorname{Tr} \rho^{2}\left(1-\operatorname{Tr} \bar{\rho}^{2}\right),
$$

where the time average over a time $T$ is defined as $\bar{f}^{T} \equiv$ $T^{-1} \int_{0}^{T} f(t) d t$. We see that large fluctuations can be achieved if there are not only large fluctuations in the energy gaps of the Hamiltonian $H_{0}$ but also if the initial state is pure enough or if the time evolution is nontrivial. If the initial state is very mixed or the time evolution does not feature an exponentially decaying Loschmidt echo, then work fluctuations will be negligible regardless of $H_{0}$.

At this point, we are ready to tackle our main goal, that is, to compute the work and its fluctuations in a quantum battery modeled by $H_{G}(t)=H_{0}+V_{G}(t)$. In this setup, one has perfect control on the measuring Hamiltonian, but the controlled quantum evolution is very noisy, as $V_{G}(t)=G^{\dagger} V(t) G$. However, one has retained control on the spectrum of the driving Hamiltonian, which is an experimentally realistic situation. In the interaction picture, and by defining $C \equiv \operatorname{Tr}\left[U_{I} \rho U_{I}^{\dagger} H_{0}\right]$, we see that work is given by

$$
\begin{aligned}
W(t) & =\operatorname{Tr}\left[\rho H_{0}\right]-\operatorname{Tr}\left[\rho_{t} H_{0}\right] \equiv E_{0}-\operatorname{Tr}\left[\rho_{t} H_{0}\right] \\
& =E_{0}-\operatorname{Tr}\left[U_{I} \rho U_{I}^{\dagger} H_{0}\right] \equiv E_{0}-C .
\end{aligned}
$$

We can write the above expression as

$$
\begin{aligned}
W(t) & =E_{0}-\operatorname{Tr}\left[U_{I} \rho \otimes U_{I}^{\dagger} H_{0} T^{(2)}\right], \\
& =E_{0}-\operatorname{Tr}\left[\left(\rho \otimes H_{0}\right)\left(U_{I} \otimes U_{I}^{\dagger}\right) T^{(2)}\right] .
\end{aligned}
$$

Now recall that the interaction picture operator $U_{I}$ depends on the random rotations $G$ as $G K G^{\dagger}$. The average work $\langle W(t)\rangle_{V}$ over the noise $G$ can then be computed (see Appendix A 1 for details) to give

$$
\langle W(t)\rangle_{V}=\left[E_{0}-\frac{\operatorname{Tr} H_{0}}{n}\right]\left[\frac{n}{n+1}+\frac{Q_{t}}{n^{2}-1}\right]
$$

with

$$
Q_{t}=-2 \sum_{j \neq k} \cos \left(\theta_{j}-\theta_{k}\right),
$$


where $\lambda_{k}=\exp \left(i \theta_{k}\right)$ are the eigenvalues of the evolution operator $K=\mathcal{T} \exp \left[-i \int_{0}^{t} V(s) d s\right]$. The time dependence of the work is thus contained in the function $Q_{t}$. For large dimension $n$, the average work reads

$$
\begin{aligned}
\langle W(t)\rangle_{V} & =\left(E_{0}-\frac{\operatorname{Tr} H_{0}}{n}\right)\left(1+\frac{Q_{t}}{n^{2}}\right) \\
& =\operatorname{Tr}\left[(\rho-\mathbb{1} / n) H_{0}\right]\left(1+Q_{t} / n^{2}\right) .
\end{aligned}
$$

At this point, averaging over the initial state $\rho$ would give zero, while averaging over the Hamiltonian $H_{0}$ gives an exponentially small work $\sim n^{-1}$.

Let us comment on the meaning of the result [Eq. (12)]. We are starting with an initial state $\rho$ and evolving with a random evolution generated by $V(t)$. So far we have averaged over rotations of the time-dependent perturbation $V_{G}(t)$. Such rotations keep the eigenvalues of $V_{G}$ unchanged so that all the results are a function of spectral quantities like $Q_{t}$. One could expect that if the evolution were completely random, then one would end up with the completely mixed state, and then the work extracted would have to be $W=\left(E_{0}-\operatorname{Tr} H_{0} / n\right)$. However, we have fixed the spectrum of $V(t)$ in the randomization, so it is remarkable that one can achieve the infinite temperature result.

Moreover, in the average work $\langle W(t)\rangle_{V}$ there is an amplifying quantum correction $\left(1+Q_{t} / n^{2}\right)$. These corrections are quantum in nature because they correspond to the constructive interference that builds up in $Q_{t}=-2 \sum_{j \neq k} \cos \left(\theta_{j}-\theta_{k}\right)$. One expects that without a specific structure in the $\theta$ 's, the factor $Q / n^{2}$ would rapidly decay to zero. This means that on average (and typically) one can achieve in this setting the same result that would be attained with random arbitrary resources. However, we can do better than that. First, if fluctuations are not a concern, then it is possible for nanosystems with small $n$ to have large $Q_{t}$. We are going to give an example in the following, using an optical cavity. Moreover, it is possible to design devices with a spectrum such that, for specific values of $t$, the term $Q_{t}$ is of order one, which can be exploited as quantum advantage in the construction of a battery. In the next section, we show how, in a specific example, revivals in $Q_{t}$ allow the battery to outperform the infinite temperature (and classical) behavior.

The question of what happens in the large- $n$ case is very interesting. In the optical cavity application shown in Sec. IV A, the quantum amplifying factor is washed out as $n^{-2}$. We think that this would happen for most models. In this sense, this is a sign of the loss of quantumness as the dimension of the Hilbert space grows. One wonders, though, whether for some specific model the amplifying factor $Q_{t} / n^{2}$ might not disappear in the large- $n$ limit. Finding such a realistic model would be of enormous practical interest. Conversely, proving that no model can feature this advantage as $n$ goes to infinity would be a very interesting result in quantum thermodynamics.

As mentioned, one expects that for a random matrix its spectrum should yield a vanishing $Q_{t}$. A natural question to ask then is what the typical behavior of this quantity is when these eigenvalues are taken randomly, according to a circulant unitary matrices (CUE) distribution (see, e.g., Ref. [32]). Let

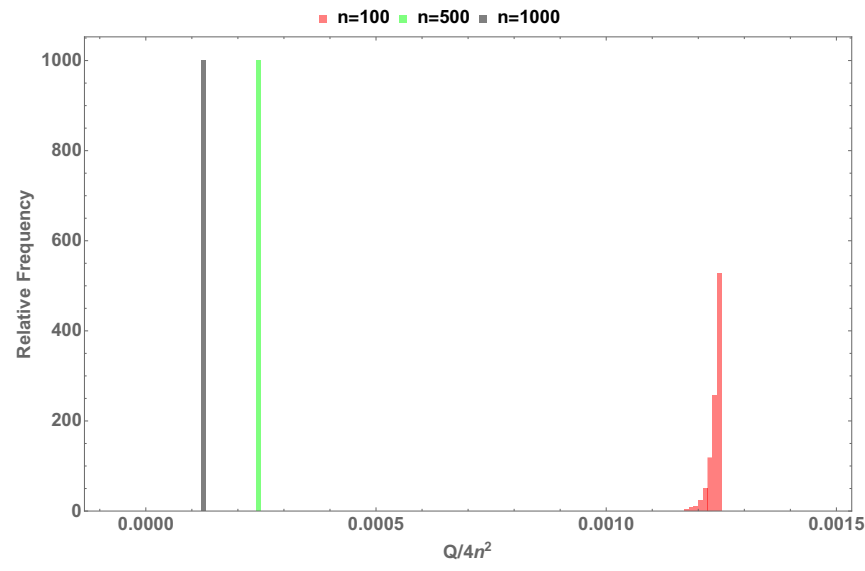

FIG. 1. Average of $Q$ over 1000 samples for random matrices in the circulant unitary ensembles of dimensions $n=10, n=$ $100,500,1000$. The peak of the distribution converges to zero for larger values of $n$.

us define $r_{k}=\lambda_{k+1} / \lambda_{k}$. We prove in Appendix B 5 that

$$
Q=\frac{1}{2} \sum_{k=1}^{n} \sum_{j=k+1}^{n}\left(\prod_{i=j+1}^{n} r_{i}+\prod_{i=j+1}^{n} r_{i}^{-1}\right) .
$$

The behavior of $Q$, evaluated numerically, is depicted in Fig. 1. We see that for large $n$ the peak of the distribution moves toward zero. That is, averaging over the spectra does not give any amplification $Q_{t}$.

How typical is the behavior of a random quantum battery in the large- $n$ limit? If there is typicality, an optimal strategy for random quantum batteries would consist in fixing the optimal spectrum of $K$ and then knowing that the other details of the evolution will not matter in the large- $n$ limit. To this end, we need to compute the fluctuations which is far more challenging because they involve the fourth tensor power of the unitary representation. We find that

$$
\Delta W_{V}^{2}=\left\langle C^{2}\right\rangle-\langle C\rangle^{2},
$$

and a lengthy calculation yields

$$
\left\langle C^{2}\right\rangle=\sum_{i} \lambda_{i} \operatorname{Tr}\left[\Pi_{i}\left(\rho \otimes H_{0}\right)^{\otimes 2}\right]
$$

with $\lambda_{i}=(\operatorname{Tr} \Pi)^{-1} \operatorname{Tr}\left(\Pi_{i} K^{\otimes 2} \otimes K^{\dagger \otimes 2}\right)$, where $\Pi$ 's are the projectors on the irreps of $S_{k}$, and the index $i$ runs over the five irreducible representations of $S_{4}$. The details of the calculation are given in Appendix B 1. Let us show that these fluctuations scale like $n^{-2}$. First, the expectation values in the above equation can be bound as

$$
\begin{aligned}
\left|\operatorname{Tr}\left[\Pi_{i}\left(\rho \otimes H_{0}\right)^{\otimes 2}\right]\right| & \leqslant\left|\operatorname{Tr}\left[\left(\rho \otimes H_{0}\right)^{\otimes 2}\right]\right|=(\operatorname{Tr} \rho)^{2}\left(\operatorname{Tr} H_{0}\right)^{2} \\
& =\left(\operatorname{Tr} H_{0}\right)^{2}=O\left(n^{2}\right) .
\end{aligned}
$$

Putting together all the terms, we find in Appendix B 2 that the fluctuations are upper bounded by

$$
\Delta W_{V}^{2} \leqslant O\left(n^{-4}\right) M(n) O\left(n^{2}\right),
$$

where $M(n)$ is an upper bound to the terms of the form $\left|\sum_{\text {mnop }} e^{i\left(\theta_{m}+\theta_{p}-\theta_{n}-\theta_{o}\right)}\right|$. If one chooses spectral properties for $K$ such that $M(n)=O(1)$, then the fluctuations scale like 
$n^{-2}$ and thus a many-body quantum battery would show exponentially small fluctuations. Moreover, this is the typical case. Indeed, by averaging over CUE to compute $M(n)$, we see in Fig. 1 that this quantity is concentrated near zero for large $n$. More in-depth numerical evidence is provided in Appendix B 2, where we analyze numerically every single term which contributes to the fluctuations, showing that indeed every single term converges to zero for large $n$ 's.

This represents the first main result of this paper: random quantum batteries show typicality in allowing a work extraction given by the difference in energy between initial state and completely mixed state, amplified (or attenued) by the form factor $\left(1+Q_{t} / n^{2}\right)$. By thus choosing a suitable $V_{0}$, one can obtain with probability almost one the desired behavior for work extraction in the sense of the Haar measure on $G V_{0} G^{\dagger}$.

\section{APPLICATIONS}

\section{A. Jaynes-Cummings model}

The specific behavior of $Q_{t}$ determines whether the quantum advantage in a random battery is washed out in the large- $n$ limit. We now apply these findings in the case of an exactly solvable model and study the behavior of $Q_{t}$. We consider a two-level system in an optical trap described by the JaynesCummings model [55]. In the rotating-wave approximation only two adjacent modes at time $(n, n+1)$ of the electromagnetic field couple with the two-level system (details provided Appendix B 3). For this calculation, we assume that the atom couples with a finite set of modes of electromagnetic field, which we truncate at a number $n=2 R$, where $R$ is a truncation of the number of modes of the electric field. At the end of the calculation we will send $R \rightarrow \infty$.

The Hamiltonian reads

$$
\begin{aligned}
H & =\omega(t) a^{\dagger} a+\frac{\Omega(t)}{2} \sigma_{z}+g(t)\left(a \sigma_{+}+a^{\dagger} \sigma_{-}\right) \\
& \equiv H_{0}+V(t)
\end{aligned}
$$

where we define $\Delta(t)=\Omega(t)-\omega(t)$, and we assume $g(t)=$ $g_{0} e^{M t}$.

For this model, we find the eigenvalues $\exp \left(i \theta_{k}\right)$ exactly and use them to evaluate Eq. (13). Following the calculation in Appendix B 3, we get $\theta_{k}-\theta_{m}=g_{0}^{2}(k-m)\left(\frac{e^{M t}-e^{M t_{0}}}{M^{2}}\right)^{2} \equiv$ $(k-m) \alpha_{t}$, where $M$ is a constant defined as $\frac{\Delta(t)}{\Delta\left(t^{\prime}\right)}=\frac{g(t)}{g\left(t^{\prime}\right)}=$ $e^{M\left(t-t^{\prime}\right)}$. We then obtain the average work Eq. (12), where, as seen above, the function $Q\left(\alpha_{t}\right)$ is a sum of trigonometric functions whose complete expression is given in Appendix B 3, Eq. (B51). We note that when $Q_{t}>0$, effectively the system extracts more work than the classical counterpark. In this sense, Fig. 2(a) shows that there can be a quantum advantage in a specific model.

In Fig. 2 we plot the time evolution of the extracted work from the random Jaynes-Cummings battery averaged over $V$. As we can see, for most times the quantum efficiency gets washed out. For small $n$, at specific revival times given by inverting Eq. (B51), the value of $Q_{t}$ becomes of order one, thus providing a nonvanishing quantum efficiency. This is at the price of performing much worse at different times. One can design a quantum battery by an array of many random nanobatteries of small $n$ and evolve to the revival time where
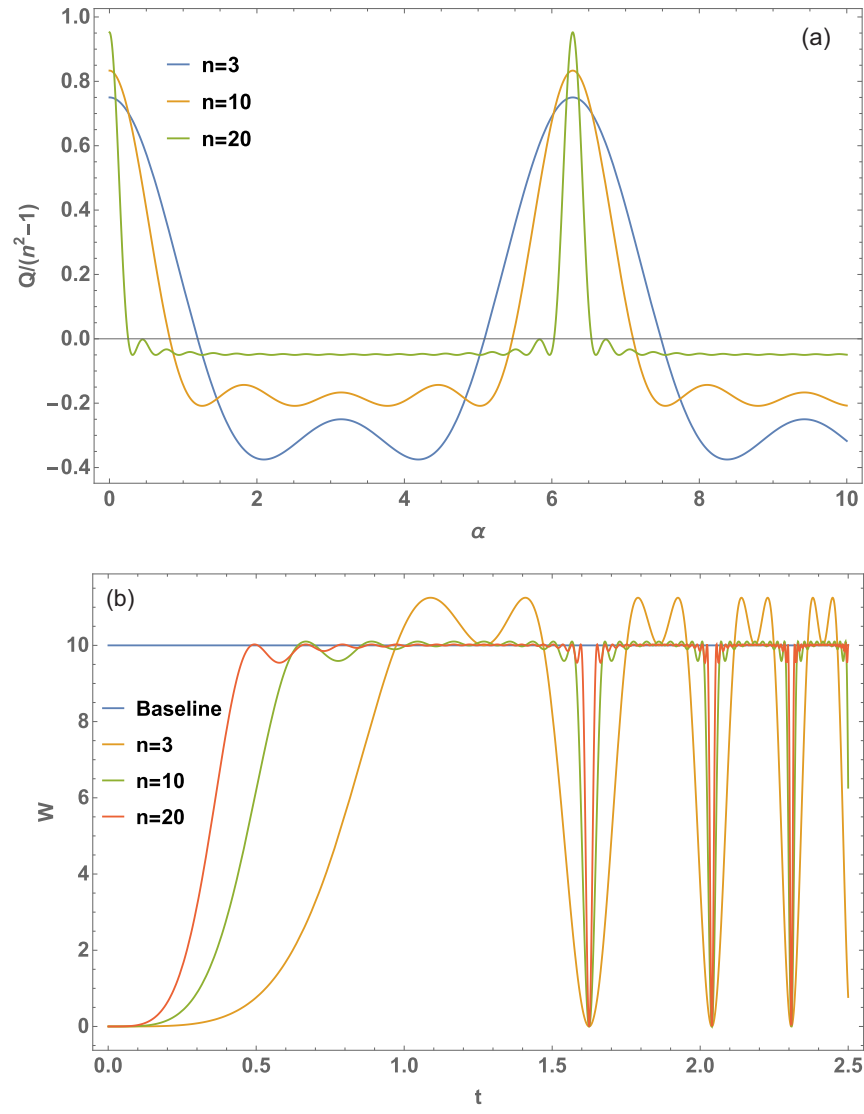

FIG. 2. Average work extraction for a random quantum battery made by an optical trap described by the Jaynes-Cummings model. (a) The function $Q(\alpha)$ as function of $\alpha$ for $n=2,10,20$. The maximum value of this function is 0.5 . As the size increases, revivals become more peaked. (b) Work for the Jaynes-Cummings model as a function of time for $\rho=0.5$ for $n=2,10,20$ and $\operatorname{Tr}\left(H_{0}\right)=90 * n$ and $E_{0}=100$. The baseline represents the work extracted by a battery that brings the system in the completely mixed state.

the work extracted goes above that corresponding to the maximally mixed state $[3,56,57]$. The fact that nonvanishing $Q_{t}$ is obtained as revivals in Eq. (13) is a sign that this amplification comes from the constructive interference coming from the complex eigenvalues of $K$ and therefore of its quantum nature. On the other hand, for large $n$, the system almost always behaves like in the limit of the battery that completely mixes the state, though one has obtained this performance with limited, realistic resources that do not require to bring the system at infinite temperature.

\section{B. Time-dependent perturbation theory}

In the case of the Jaynes-Cummings model we could solve for the time evolution exactly, finding expressions for the average work and its fluctuations via perturbation theory. We make use of the Dyson series for the evolution operator in the interaction picture, namely $U_{I}(t)=\mathcal{T} \sum_{n=0}^{\infty} \frac{(-i)^{n}}{n !}\left[\int_{0}^{t} d t^{\prime} V_{I}\left(t^{\prime}\right)\right]^{n}$. We consider perturbations up to the second order in the Dyson series, and at this point we can average over $G$. Define the operator $A=\int_{t_{0}}^{t} V_{0}\left(t^{\prime}\right) d t^{\prime}$. Again we need the fluctuations of 


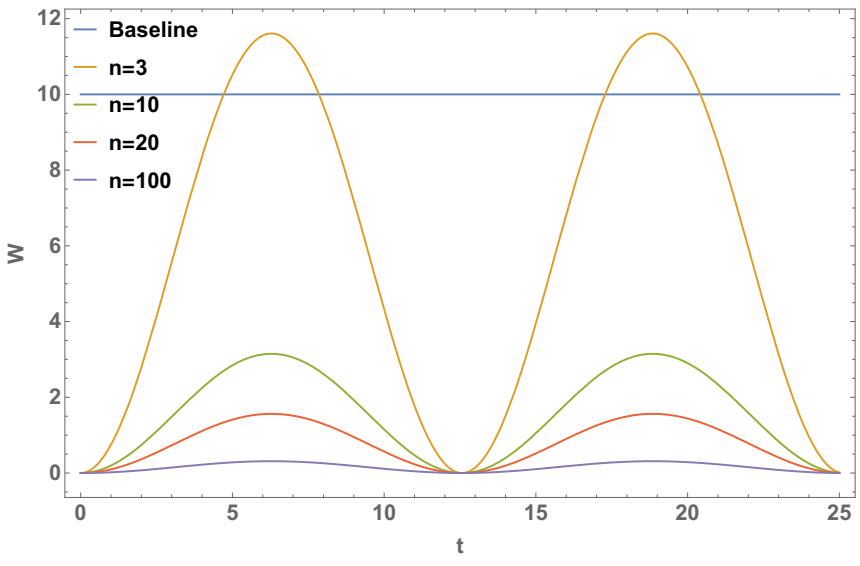

FIG. 3. Average work from Eq. (B74) for $n=3,10,20,100$ and $\omega=0.5$, against the baseline work $E_{0}-\frac{\operatorname{Tr}\left(H_{0}\right)}{n}$, with $\operatorname{Tr}\left(H_{0}\right)=90 n$ and $E_{0}=100$.

$A$ in the completely mixed state, namely $n^{2} \Delta A^{2}=n \operatorname{Tr} A^{2}-$ $(\operatorname{Tr} A)^{2}$.

Averaging over $G$ requires a lengthy calculation (see Appendix B 4) yielding

$$
\langle W(t)\rangle_{V}=\frac{n^{2} \Delta A^{2}}{n^{2}-1}\left[E_{0}-\frac{\operatorname{Tr}\left(H_{0}\right)}{n}\right] .
$$

The second term is the difference between the initial energy and the energy in the completely mixed state.

As an example consider the case of an exactly solvable Hamiltonian $H_{0}$ subject to the harmonic perturbation $V(t)=$ $\hat{V}_{0} e^{i \omega t}+\hat{V}_{0}^{\dagger} e^{-i \omega t}$. Let $\lambda_{k}$ be the eigenvalues of $\hat{V}_{0}$. Averaging over $V$ results in

$$
\begin{aligned}
\langle W(t)\rangle_{V}= & \frac{2 f^{2}(t, \omega)}{\left(n^{2}-1\right)}\left\{\sum_{k, k^{\prime}} \operatorname{Re}\left(\lambda_{k} e^{i \frac{t+t_{0}}{2} \omega}\right) \operatorname{Re}\left(\lambda_{k^{\prime}} e^{i \frac{t+t_{0}}{2} \omega}\right)\right. \\
& \left.-2 n \sum_{k} \operatorname{Re}\left[\lambda_{k}^{2} e^{i \omega\left(t_{0}+t\right)}\right]+2 n \sum_{k} \sigma_{k}\right\},
\end{aligned}
$$

where we have defined $f(t, \omega)=2 \sin \left(\frac{t-t_{0}}{2} \omega\right) / \omega$ and $\lambda$ 's are the eigenvalues of $\hat{V}$. As one can see, the average work decreases with $n$. We plot $\langle W(t)\rangle_{V}$ in Fig. 3. In this model it is easy to find the revival times at which the quantum efficiency is maintained also for larger values of $n$. One can indeed show (see Appendix B 4) that the work performed by a random harmonic perturbation of the form $2 \hat{V} \cos (\omega t)$ has always a single maximum at $t_{k}=(2 k+1) \frac{\pi}{\omega}$ on average.

\section{Adiabatic quantum batteries}

Now let us consider the case of a quantum battery performing an adiabatic evolution connecting the two Hamiltonians $H_{0}$ and $H_{1}$ and the two respective equilibrium states $\rho_{0}, \rho_{1}$, e.g., two eigenstates or Gibbs states for $H_{0}, H_{1}$ (but also thermal or more general mixed equilibrium states). Adiabatic evolution as a method to perform quantum computation [58] or quantum control has been long an important tool in quantum information processing, see, e.g., Ref. [59]. Adiabatic evolution to perform work extraction was studied in Ref. [60].
A model for an adiabatic quantum battery based on a threelevel system was studied in Ref. [61]. In this section, we deal with general adiabatic quantum batteries in which the adiabatic drive is rotated in a random direction as a function of time.

In general, two Hamiltonians are adiabatically connectible if and only if they belong to the same connected component of the set of isodegenerate Hamiltonians [62]. By denoting $H_{\alpha}=\sum_{i=1}^{R} \epsilon_{\alpha}^{i} \Pi_{\alpha}^{i}(\alpha=0,1)$ the spectral resolution of $H_{0}$ and $H_{1}$, and ordering their eigenvalues in ascending order, i.e., $\epsilon_{\alpha}^{1}<\cdots<\epsilon_{\alpha}^{R}$, we define the vectors $D_{\alpha}:=$ $\left(\operatorname{tr} \Pi_{\alpha}^{1}, \ldots, \Pi_{\alpha}^{R}\right) \equiv\left(d_{\alpha}^{1} \ldots d_{\alpha}^{R}\right)$, with $\sum_{k} d_{\alpha}^{k}=n$. The Hamiltonians $H_{0}$ and $H_{1}$ belong to the same connected component of the set of isodegenerate Hamiltonians iff $D_{0}=D_{1}$. So, speaking of adiabatically connected Hamiltonians, we can drop the index $\alpha$. Let us now introduce the functions $\epsilon^{i}:[0,1] \mapsto \mathbb{R}$ such that $\epsilon^{i}(0)=\epsilon_{0}^{i}$, and $\epsilon_{i}(1)=\epsilon_{1}^{i}((i=1, \ldots, R)$ obeying the no-crossing condition $\epsilon^{i+1}(t)>\epsilon^{i}(t)(i=1, \ldots, R-1)$. A continuous family of Hamiltonians connecting $H_{0}, H_{1}$ has then the form $H(t)=\sum_{i=1}^{R} \epsilon^{i}(t) U_{t} \Pi_{0}^{i} U_{t}^{\dagger}$, where the continuous unitary family $\left\{U_{t}\right\}_{t=0}^{1}$ is such that $U_{0}=\mathbb{1}$ and $U_{1}=U$. The work extracted after the adiabatic evolution thus reads

$$
\begin{aligned}
W & =\operatorname{Tr}\left(\rho_{0} H_{0}\right)-\operatorname{Tr}\left(\rho_{1} H_{0}\right) \\
& =\sum_{i=1}^{R} \operatorname{Tr}\left[p_{i}\left(\Pi_{0}^{i}-\Pi_{1}^{i}\right) H_{0}\right] \\
& =\sum_{i, j=1}^{R} p_{i} \epsilon_{0}^{j} \operatorname{Tr}\left[\left(\Pi_{0}^{i}-\Pi_{1}^{i}\right) \Pi_{0}^{j}\right],
\end{aligned}
$$

because the populations in the $i$ th subspace are conserved by the adiabatic evolution. We now have $\Pi_{\alpha}^{i} \Pi_{\beta}^{j}=\delta^{i j}$ if $\alpha=\beta$, but otherwise they are not necessarily orthogonal. We see that the work depends on the choice of $U$ as

$$
\begin{aligned}
W_{U} & =\sum_{i j} p_{i} \epsilon_{0}^{j}\left[\operatorname{Tr}\left(\Pi_{0}^{i} \Pi_{0}^{j}\right)-\operatorname{Tr}\left(\Pi_{0}^{i} \Pi_{1}^{j}\right)\right] \\
& =\sum_{i j} p_{i} \epsilon_{0}^{j}\left[d_{i} \delta_{i j}-\operatorname{Tr}\left(\Pi_{0}^{i} U \Pi_{0}^{j} U^{\dagger}\right)\right] .
\end{aligned}
$$

We can now perform the average over the unitary transformation $U$. We easily obtain

$$
\begin{aligned}
\langle W\rangle_{a d} & =\sum_{i j} p_{i} \epsilon_{0}^{j}\left[d_{i} \delta_{i j}-\operatorname{Tr}\left(\Pi_{0}^{i} \frac{d_{j} \mathbb{I}}{n}\right)\right] \\
& =\sum_{i j} p_{i} \epsilon_{0}^{j}\left(d_{i} \delta_{i j}-\frac{d_{i} d_{j}}{n}\right), \\
& =E_{0}-\sum_{i j} p_{i} \epsilon_{0}^{j} \frac{d_{i} d_{j}}{n} .
\end{aligned}
$$

To understand the role of the degeneracies, let us consider the case of a nondegenerate Hamiltonian, so that $d_{i}=1$ for all $i$. We obtain $\langle W\rangle_{a d}=E_{0}-\operatorname{Tr} H_{0} / n$, which again is the difference between the initial energy and the energy of the completely mixed state and thus the quantum efficiency is washed out (see Ref. [63]). More generally, as we show in 
Appendix B 6, we find an upper bound on the adiabatic work given by

$$
\begin{gathered}
\langle W\rangle_{a d} \leqslant E_{0}(1+c)-\frac{\operatorname{Tr}\left(H_{0}\right)}{n}, \\
c=\frac{\sum_{i} d_{i}^{2}-n}{n},
\end{gathered}
$$

so that potentially random adiabatic quantum batteries could give an advantage over classical devices as well (even at infinite temperature), as $c \geqslant 0$.

Let us now look at the fluctuations $\Delta W_{a d}^{2}$. The calculation involves averaging the square of the work and thus the order two tensored representation of the unitary group. This is also a lengthy calculation, whose details are given in Appendix B 6. We obtain

$$
\begin{aligned}
\Delta W_{a d}^{2}= & \sum_{i, j, k, l} p_{i} \epsilon_{0}^{j} p_{k} \epsilon_{0}^{l}\left[\frac{d_{i} d_{j} d_{k} d_{l}}{n^{2}-1}-\frac{d_{i} d_{k} d_{l} \delta_{l j}}{n\left(n^{2}-1\right)}\right. \\
& \left.-\frac{d_{j} d_{k} d_{l} \delta_{k i}}{n\left(n^{2}-1\right)}+\frac{d_{k} d_{l} \delta_{k i} \delta_{l j}}{n^{2}-1}-\frac{d_{i} d_{j} d_{k} d_{l}}{n^{2}}\right] .
\end{aligned}
$$

For $n \gg 1$, the terms of order $1 / n^{3}$ go to zero faster than $1 / n^{2}$, and we obtain

$$
\begin{aligned}
\Delta W_{a d}^{2} \underbrace{=}_{n \gg 1} \frac{1}{n^{2}} \sum_{i, j, k, l} p_{i} \epsilon_{0}^{j} p_{k} \epsilon_{0}^{l}\left(d_{k} d_{l} \delta_{k i} \delta_{l j}\right) \\
=\frac{\operatorname{Tr}\left(H_{0} \rho_{0}\right)^{2}}{n^{2}}=\frac{E_{0}^{2}}{n^{2}},
\end{aligned}
$$

which shows that random adiabatic quantum batteries feature typicality. Fluctuations during adiabatic driving were studied in a different context also in Ref. [64].

\section{CONCLUSIONS AND OUTLOOK}

In this paper we provided a notion of quantum random batteries by means of Haar averaging initial states, energy measurement Hamiltonian, and the time-dependent driving of the quantum battery. This method allows to study large classes of systems, including not-exactly solvable systems or adiabatic quantum batteries. The average work and fluctuations are systematically studied; we find that quantum batteries exhibit typical behavior in the large- $n$ limit given the spectral properties of the driving system. On average, the work extracted is found to be typically equal to the difference between the energy of the initial state and that of the completely mixed state, amplified by a quantum efficiency factor $\left(1+Q_{t} / n^{2}\right)$ that only depends on the spectrum of the driving Hamiltonian. Quantum efficiency is not washed out at specific revival times for small systems. Our method allows for the computation of $Q_{t}$ in perturbation theory, therefore allowing for the treatment of realistic systems. We have also treated the case of random adiabatic quantum batteries, finding that amplification is lost for a nondegenerate Hamiltonian.

In perspective, our results put forward several questions that we would like to investigate in the immediate future. We have shown that for small systems there are revival times in which quantum coherence builds up and gives a quantum advantage. Typically, this is not the case for large $n$. However, it is an open problem whether there are random quantum batteries whose spectral properties allow for the build-up of coherence that outperforms the classical case. Conversely, showing the impossibility of such quantum amplification for large $n$ would be an important result in quantum thermodynamics. This is a problem which we plan to explore in the near future in a realistic model. A second question relating to the effect of quantum coherence also arises. As we have seen, the extracted work can be related to the coherence of the initial state in two different bases or of the operator $U_{t}$ in two different bases. This suggests that there is a nontrivial interplay between coherence and work that involves more than one basis [65]. Also, the lack of commutativity between the initial state and the evolution operator or the measuring Hamiltonian and the evolution operator take the form of out-of-time-order correlators (OTOC). It would then be interesting to explore the connection among fast decays of these quantities, chaos, scrambling, and work statistics. One very intriguing insight comes from the fact that the narrowing of fluctuations does shrink the quantum efficiency but at specific revival times. These revival times correspond to spectral properties of the time evolution operator and one would be interested in understanding the connection between quantum efficiency of random quantum batteries and the integrability or chaotic behavior of the Hamiltonian. Using tools from local Haar averaging [40], we can explore whether the efficiency in a battery with a microscopic local drive is influenced by quantum chaos or integrability. The optimization of the path in a adiabatic quantum algorithm is related to the brachistochrone or geodesics in the space of the ground-state manifold [66]. It would be very interesting to see whether optimal paths correspond to bounds given by quantum thermodynamics. Finally, it would be important to generalize these results to the case of open quantum systems.

\section{ACKNOWLEDGMENTS}

The work of F.C. was carried out under the auspices of the NNSA of the US DoE at LANL under Contract No. DE-AC52-06NA25396. F.C. was also financed via DOE-ER Grants No. PRD20170660 and No. PRD20190195. L.P.G.-P. also acknowledges partial support by DoE ASCR Quantum Testbed Pathfinder program (Award No. DE- sc0019040), ARO MURI, NSF PFCQC program, ARL CDQI, AFOSR, DoE BES QIS program (Award No. DE-sc0019449), and NSF PFC at JQI. A.H. thanks Robert Whitney for insightful conversations at LPMMC, Grenoble, France.

\section{APPENDIX A}

\section{Calculation of $\Delta W_{\rho}^{2}$}

Since $W=0$ and $W^{2}=\operatorname{Tr} W \cdot \operatorname{Tr} W=\operatorname{Tr}(W \otimes W)$, we see that $\Delta W^{2}=\left\langle W^{2}\right\rangle=\left\langle\operatorname{Tr}\left(\rho \delta H_{0}\right)^{\otimes 2}\right\rangle=\operatorname{Tr}\left[\left\langle\rho^{\otimes 2}\right\rangle \delta H^{\otimes 2}\right]$. The average on the tensored representation $G^{\otimes 2} \rho^{\otimes 2} G^{\dagger \otimes 2}$ is also well known [40,41] and is the linear combination on the irreps of $S_{2}$ given by $\left\langle\rho^{\otimes 2}\right\rangle=\sum_{ \pm} \lambda_{ \pm} \Pi_{ \pm}$with $\lambda_{ \pm}=$ $\operatorname{Tr}\left(\Pi_{ \pm} \rho^{\otimes 2}\right) / \operatorname{Tr} \Pi_{ \pm}$and $\Pi_{ \pm}=\left(\mathbb{1}^{\otimes 2}+T^{(2)}\right) / 2$, where $\left.T^{(2)}\right)$ is the order two permutation ("swap") operator on $\mathcal{H}^{\otimes 2}$. Thus 
we obtain

$$
\begin{aligned}
\Delta W_{\rho}^{2} & =\sum_{ \pm} \lambda_{ \pm} \operatorname{Tr}\left(\Pi_{ \pm} \delta H^{\otimes 2}\right) \\
& =\frac{\left[\left(\lambda_{+}+\lambda_{-}\right) \operatorname{Tr} \delta H^{\otimes 2}+\left(\lambda_{+}-\lambda_{-}\right) \operatorname{Tr}\left(T^{(2)} \delta H^{\otimes 2}\right)\right]}{2} \\
& =\frac{\left(\lambda_{+}+\lambda_{-}\right)(\operatorname{Tr} \delta H)^{2}+\frac{1}{2}\left(\lambda_{+}-\lambda_{-}\right) \operatorname{Tr} \delta H^{2}}{2} .
\end{aligned}
$$

We saw above that $\operatorname{Tr} \delta H=0$ and finally we obtain

$$
\Delta W_{\rho}^{2}=\frac{n \operatorname{Tr} \rho^{2}-1}{n\left(n^{2}-1\right)} \operatorname{Tr} \delta H^{2}=2 \frac{n \operatorname{Tr} \rho^{2}-1}{n\left(n^{2}-1\right)} \operatorname{Tr} H_{0}^{2},
$$

which is the result we present in the paper.

\section{Work fluctuations averaging on $\boldsymbol{H}_{0}$}

Let us define $R=\rho-\rho_{t}$. We consider the fluctuations on the work via the averaging on the operator $H_{0}$. We have

$$
\begin{aligned}
\Delta W_{H_{0}}^{2} & =\left\langle W^{2}\right\rangle=\operatorname{Tr}\left[R^{\otimes 2} \sum_{ \pm} \lambda_{ \pm} \Pi_{ \pm}\right] \\
& =\frac{1}{2} \sum_{ \pm} \lambda_{ \pm} \operatorname{Tr}\left[R^{\otimes 2} \pm T^{(2)} R^{\otimes 2}\right] \\
& =\frac{1}{2} \sum_{ \pm} \lambda_{ \pm}\left[(\operatorname{Tr} R)^{2} \pm \operatorname{Tr} R^{2}\right] \\
& =\frac{1}{2}\left(\lambda_{+}-\lambda_{-}\right) \operatorname{Tr} R^{2},
\end{aligned}
$$

where now the coefficients of the projectors are $\lambda_{ \pm}=$ $\operatorname{Tr}\left(\Pi_{ \pm} H_{0}^{\otimes 2}\right) / \operatorname{Tr} \Pi_{ \pm}$. Direct calculation gives, defining $a \equiv$ $\left(\operatorname{Tr} H_{0}\right)^{2}$ and $b \equiv \operatorname{Tr} H_{0}^{2}$,

$$
\begin{aligned}
\frac{1}{2}\left(\lambda_{+}-\lambda_{-}\right)= & \frac{1}{n(n+1)}(a+b)-\frac{1}{n(n-1)}(a-b), \\
= & {\left[\frac{1}{n(n+1)}-\frac{1}{n(n-1)}\right] a } \\
& +\left[\frac{1}{n(n+1)}-\frac{1}{n(n-1)}\right] b, \\
= & \frac{2}{n\left(n^{2}-1\right)}(n b-a) .
\end{aligned}
$$

The work fluctuations can thus be written as

$$
\Delta W_{H_{0}}^{2}=\frac{2}{n\left(n^{2}-1\right)}\left[n \operatorname{Tr} H_{0}^{2}-\left(\operatorname{Tr} H_{0}\right)^{2}\right] \operatorname{Tr} R^{2} .
$$

Now consider the fluctuations $\Delta H_{0}^{2}$ of the eigenvalues of the Hamiltonian $H_{0}$, namely the fluctuations of $H_{0}$ in the completely mixed state $11 / n$. We have

$$
\Delta H_{0}^{2}=\frac{1}{n} \operatorname{Tr} H_{0}^{2}-\frac{1}{n^{2}}\left(\operatorname{Tr} H_{0}\right)^{2},
$$

we then obtain

$$
\Delta W_{H_{0}}^{2}=\frac{2}{n\left(n^{2}-1\right)} n^{2} \Delta H_{0}^{2} \operatorname{Tr} R^{2},
$$

and thus, finally,

$$
\begin{aligned}
\Delta W_{H_{0}}^{2} & =\left\langle W^{2}\right\rangle_{H_{0}}=\operatorname{Tr}\left[\left(\rho-\rho_{t}\right)^{\otimes 2} \sum_{ \pm} \lambda_{ \pm} \Pi_{ \pm}\right] \\
& =\frac{2 n}{n^{2}-1} \Delta H_{0}^{2} \operatorname{Tr}\left(\rho^{2}-\rho \rho_{t}\right),
\end{aligned}
$$

which is the result we report in the paper.

\section{Traces of $K$}

A direct calculation of the coefficients yields

$$
\begin{aligned}
& \lambda_{+}=\frac{\operatorname{Tr}\left(K \otimes K^{\dagger} \Pi_{+}\right)}{\operatorname{Tr} \Pi_{+}}=\frac{2}{n(n+1)} \frac{\operatorname{Tr} K \operatorname{Tr} K^{\dagger}+\operatorname{Tr} K K^{\dagger}}{2} \\
& \lambda_{-}=\frac{\operatorname{Tr}\left(U_{0} \otimes U_{0}^{\dagger} \Pi_{-}\right)}{\operatorname{Tr} \Pi_{-}}=\frac{2}{n(n-1)} \frac{\operatorname{Tr} K \operatorname{Tr} K^{\dagger}-\operatorname{Tr} K K^{\dagger}}{2} .
\end{aligned}
$$

Moreover, we use that

$$
\lambda_{+} \Pi_{+}+\lambda_{-} \Pi_{-}=\frac{\lambda_{+}+\lambda_{-}}{2} \mathbb{I}+\frac{\lambda_{+}-\lambda_{-}}{2} T .
$$

We now see that, defining

$$
\begin{aligned}
& a=\operatorname{Tr} K \operatorname{Tr} K^{\dagger}=\left|\sum_{i} e^{i \theta_{i}}\right|^{2}=2 \sum_{j \neq k} \cos \left(\theta_{j}-\theta_{k}\right)+n \\
& b=\operatorname{Tr} K K^{\dagger}=n
\end{aligned}
$$

and thus

$$
\lambda_{+}=\frac{1}{n} \frac{a+b}{n+1}, \quad \lambda_{-}=\frac{1}{n} \frac{a-b}{n-1},
$$

and using the relationships

$$
\begin{aligned}
& \frac{1}{2 n}\left[\frac{a+b}{(n+1)}+\frac{a-b}{(n-1)}\right]=\frac{a n-b}{n^{3}-n} \\
& \frac{1}{2 n}\left[\frac{a+b}{(n+1)}-\frac{a-b}{(n-1)}\right]=\frac{b n-a}{n^{3}-n},
\end{aligned}
$$

we get

$$
\begin{aligned}
\frac{\lambda_{+}+\lambda_{-}}{2} & =\frac{n\left[2 \sum_{j \neq k} \cos \left(\theta_{j}-\theta_{k}\right)+n\right]-n}{n^{3}-n} \\
& =\frac{2 \sum_{j \neq k} \cos \left(\theta_{j}-\theta_{k}\right)+n-1}{n^{2}-1} \\
\frac{\lambda_{+}-\lambda_{-}}{2} & =\frac{n^{2}-\left[2 \sum_{j \neq k} \cos \left(\theta_{j}-\theta_{k}\right)+n\right]}{n\left(n^{2}-1\right)} .
\end{aligned}
$$

\section{Calculation of $\langle W(t)\rangle_{V}$ and $\Delta W_{V}^{2}$}

The work extracted $W(t)$ reads

$$
\begin{aligned}
W(t) & =\operatorname{Tr}\left[\omega H_{0}\right]=\operatorname{Tr}\left[\rho H_{0}\right]-\operatorname{Tr}\left[\rho_{t} H_{0}\right] \equiv E_{0}-\operatorname{Tr}\left[\rho_{t} H_{0}\right] \\
& =E_{0}-\operatorname{Tr}\left[U_{I} \rho U_{I}^{\dagger} H_{0}\right] \equiv E_{0}-C .
\end{aligned}
$$

We can write the above expression as

$$
\begin{aligned}
W(t) & =E_{0}-\operatorname{Tr}\left[U_{I} \rho \otimes U_{I}^{\dagger} H_{0} T^{(2)}\right], \\
& =E_{0}-\operatorname{Tr}\left[\left(\rho \otimes H_{0}\right)\left(U_{I} \otimes U_{I}^{\dagger}\right) T^{(2)}\right] .
\end{aligned}
$$


The average work over the noise $G$ can then be computed as

$$
\begin{aligned}
\langle W(t)\rangle_{V} & =E_{0}-\operatorname{Tr}\left[\left(\rho \otimes H_{0}\right)\left\langle\left(U_{I} \otimes U_{I}^{\dagger}\right)\right\rangle T^{(2)}\right] \\
& =E_{0}-\operatorname{Tr}\left[\left(\rho \otimes H_{0}\right)\left\langle\left(G K G^{\dagger} \otimes G K^{\dagger} G^{\dagger}\right)\right\rangle T^{(2)}\right] \\
& =E_{0}-\operatorname{Tr}\left[\left(\rho \otimes H_{0}\right)\left\langle G^{\otimes 2}\left(K \otimes K^{\dagger}\right) G^{\dagger \otimes 2}\right\rangle T^{(2)}\right] .
\end{aligned}
$$

The unitary operator $K=\mathcal{T} \exp \left[-i \int_{0}^{t} V(s) d s\right]$ will be diagonalized in the form $K=\sum_{k} \exp \left(i \theta_{k}\right)|k\rangle\langle k|$.

Using the usual technique, we find $\left\langle G^{\otimes 2}\left(K \otimes K^{\dagger}\right) G^{\dagger \otimes 2}\right\rangle=$ $\sum_{ \pm} \lambda_{ \pm} \Pi_{ \pm}$, where now $\lambda_{ \pm}=\operatorname{Tr}\left(\Pi_{ \pm} K \otimes K^{\dagger}\right) / \operatorname{Tr} \Pi_{ \pm}$. Notice that in this setup, already the average work involves the average over the tensored representation of the unitary group. We obtain

$$
\begin{aligned}
& \langle W(t)\rangle_{V} \\
& =E_{0}-\operatorname{Tr}\left[\left(\frac{\lambda_{+}+\lambda_{-}}{2} \mathbb{I}+\frac{\lambda_{+}-\lambda_{-}}{2} T\right) T^{(2)}\left(\rho_{0} \otimes H_{0}\right)\right] \\
& =E_{0}-\operatorname{Tr}\left[\left(\frac{\lambda_{+}+\lambda_{-}}{2} T^{(2)}+\frac{\lambda_{+}-\lambda_{-}}{2} \mathbb{I}\right)\left(\rho_{0} \otimes H_{0}\right)\right] \\
& =E_{0}\left(1-\frac{\lambda_{+}+\lambda_{-}}{2}\right)-\frac{\lambda_{+}-\lambda_{-}}{2} \operatorname{Tr}\left(\rho_{0}\right) \operatorname{Tr}\left(H_{0}\right) \\
& =E_{0}\left[1-\frac{2 \sum_{j \neq k} \cos \left(\theta_{j}-\theta_{k}\right)+n-1}{n^{2}-1}\right] \\
& \quad-\frac{n^{2}-\left[2 \sum_{j \neq k} \cos \left(\theta_{j}-\theta_{k}\right)+n\right] \frac{\operatorname{Tr}\left(H_{0}\right)}{n} .}{n^{2}-1} .
\end{aligned}
$$

We finally obtain

$$
\langle W(t)\rangle_{V}=\left[E_{0}-\frac{\operatorname{Tr} H_{0}}{n}\right]\left[\frac{n}{n+1}+\frac{Q}{n^{2}-1}\right],
$$

where in the above equation, $\exp \left(i \theta_{k}\right)$ are the eigenvalues of the evolution operator $K=\mathcal{T} \exp \left[-i \int_{0}^{t} V(s) d s\right]$. All the time dependence of the is thus contained in the function $Q\left(\theta_{j}-\theta_{k}\right)$.

The fluctuations are more challenging because they involve the fourth tensor power of the unitary representation. Let us set out to find them. We see that

$$
\Delta W_{V}^{2}=\left\langle C^{2}\right\rangle-\langle C\rangle^{2},
$$

where $C \equiv \operatorname{Tr}\left[U_{I} \rho U_{I}^{\dagger} H_{0}\right]$. The relevant object to compute is then

$$
\begin{aligned}
\left\langle C^{2}\right\rangle & =\operatorname{Tr}\left[\left\langle\left(U_{I} \rho U_{I}^{\dagger}\right)^{\otimes 2}\right\rangle H_{0}^{\otimes 2}\right], \\
& =\operatorname{Tr}\left[\left\langle\left(G K G^{\dagger} \rho G K^{\dagger} G\right)^{\otimes 2}\right\rangle H_{0}^{\otimes 2}\right], \\
& =\operatorname{Tr}\left[\left\langle G^{\otimes 4}\left(K \otimes K^{\dagger}\right)^{\otimes 2} G^{\dagger \otimes 4}\right\rangle\left(\rho \otimes H_{0}\right)^{\otimes 2}\right] .
\end{aligned}
$$

This time, the average reads

$$
\left\langle G^{\otimes 4}\left(K \otimes K^{\dagger}\right)^{\otimes 2} G^{\dagger \otimes 4}\right\rangle=\sum_{i} \lambda_{i} \Pi_{i}
$$

with $\lambda_{i}=(\operatorname{Tr} \Pi)^{-1} \operatorname{Tr}\left(\Pi_{i} K^{\otimes 2} \otimes K^{\dagger \otimes 2}\right)$. Now the $\Pi_{i}$ are the projectors onto the irreps of $S_{4}$. There are five irreducible irreps of $S_{4}$. In the next subsection we show an explicit
TABLE I. The character table of $S_{4}$.

\begin{tabular}{lrrrrr}
\hline \hline & $e$ & $(12)$ & $(123)$ & $(1234)$ & $(12)(34)$ \\
\hline trivial & 1 & 1 & 1 & 1 & 1 \\
sgn & 1 & -1 & 1 & -1 & 1 \\
st & 3 & 1 & 0 & -1 & -1 \\
st $\otimes$ sgn & 3 & -1 & 0 & 1 & -1 \\
2D & 2 & 0 & -1 & 0 & 2 \\
size & 1 & 6 & 8 & 6 & 3 \\
\hline \hline
\end{tabular}

expression of these projectors. A lengthy calculation yields

$$
\begin{aligned}
\left\langle C^{2}\right\rangle= & \sum_{i} \lambda_{i} \operatorname{Tr}\left[\Pi_{i}\left(\rho \otimes H_{0}\right)^{\otimes 2}\right] \\
= & \frac{\operatorname{Tr}\left[\Pi^{(\operatorname{tr})}\left(\rho \otimes H_{0}\right)^{\otimes 2}\right]}{\operatorname{Tr}\left[\Pi^{(\mathrm{tr})}\right]}+\frac{\operatorname{Tr}\left[\Pi^{(\mathrm{sig})}\left(\rho \otimes H_{0}\right)^{\otimes 2}\right]}{\operatorname{Tr}\left[\Pi^{(\mathrm{sig})}\left(\rho \otimes H_{0}\right)^{\otimes 2}\right]} \\
& +\frac{\operatorname{Tr}\left[\Pi^{(\mathrm{st})}\left(\rho \otimes H_{0}\right)^{\otimes 2}\right]}{\operatorname{Tr}\left[\Pi^{(\mathrm{st})}\right]}+\frac{\operatorname{Tr}\left[\Pi^{(\mathrm{st} \otimes \mathrm{sgn})}\left(\rho \otimes H_{0}\right)^{\otimes 2}\right]}{\operatorname{Tr}\left[\Pi^{(\mathrm{st} \otimes \mathrm{sgn})}\right]} \\
& +\frac{\operatorname{Tr}\left[\Pi^{(2 \mathrm{D})}\left(\rho \otimes H_{0}\right)^{\otimes 2}\right]}{\operatorname{Tr}\left[\Pi^{(2 \mathrm{D})}\right]} .
\end{aligned}
$$

\section{Irreps of $S_{4}$}

Let us first recall the character table of $S_{4}$ in Table I. The last row of Table I gives the size of each conjugacy class in $S_{4}$. Given a permutation $\sigma \in S_{4}$, we denote by $S(\sigma)$ the representation of $S: S_{4} \mapsto G L\left(\mathcal{H}^{\otimes 4}\right)$ given by

$$
S(\sigma)=\sum_{i j k l}|\sigma(i j k l)\rangle\langle i j k l| .
$$

By the Schur-Weyl duality the projectors onto its irreps are

$$
\Pi^{(r)}=\frac{\chi^{(r)}(e)}{\left|S_{4}\right|} \sum_{\sigma \in S_{4}} \chi^{(r)}(\sigma) S(\sigma),
$$

where $\chi^{(r)}$ is the character of the $(r)$ irrep of $S_{4}$ and $\chi^{(e)}$ is the dimension of the irrep in $S_{4}$.

The five projectors are given by:

$$
\begin{aligned}
\Pi^{(\mathrm{tr})}= & \frac{1}{24} \sum_{S_{4}} S(\sigma) \\
\Pi^{(\mathrm{sig})}= & \frac{1}{24}\left[\sum_{\sigma \text { even }} S\left(\sigma_{\mathrm{ev}}\right)-\sum_{\sigma \text { odd }} S\left(\sigma_{\text {odd }}\right)\right] \\
\Pi^{(\mathrm{st})}= & \frac{3}{24}\left\{3 \mathbb{1}+\left[S_{(12)}+\cdots\right]-\left[S_{(1234)}+\cdots\right]\right. \\
& \left.-\left[S_{(12)(34)}+\cdots\right]\right\} \\
\Pi^{(\mathrm{st} \otimes \mathrm{sgn})}= & \frac{3}{24}\left\{3 \mathbb{1}-\left[S_{(12)}+\cdots\right]+\left[S_{(1234)}+\cdots\right]\right. \\
& \left.-\left[S_{(12)(34)}+\cdots\right]\right\} \\
\Pi^{(2 \mathrm{D})}= & \frac{2}{24}\left\{2 \mathbb{1}-\left[S_{(123)}+\cdots\right]+2\left[S_{(12)(34)}+\cdots\right]\right\} .
\end{aligned}
$$

In the above, the symbol $+\cdots$ denotes a sum over all the members of the conjugacy class. As is well known, the five conjugacy classes of $S_{4}$ are given by their cycle structure of Table II. 
TABLE II. The conjugacy classes of $S_{4}$ which we use for the projectors.

\begin{tabular}{llccc}
\hline \hline$e$ & $(.)$. & $(.).(.)$. & $(\ldots)$ & $(\ldots)$. \\
\hline$e$ & $(12)$ & $(12)(34)$ & $(123)$ & $(1234)$ \\
& $(13)$ & $(13)(24)$ & $(132)$ & $(1342)$ \\
& $(14)$ & $(14)(23)$ & $(124)$ & $(1423)$ \\
& $(23)$ & & $(142)$ & $(1243)$ \\
& $(24)$ & & $(134)$ & $(1432)$ \\
& $(34)$ & & $(143)$ & $(1324)$ \\
& & $(234)$ & \\
& & $(243)$ & \\
\hline \hline
\end{tabular}

\section{APPENDIX B: WORK FLUCTUATIONS VIA IRREPS OF $S_{4}$}

\section{Main definitions and projectors}

Before we begin the calculation, we start with a few definitions which will be useful in the following:

$$
\begin{aligned}
K & =\sum_{k} e^{i \theta_{k}}|k\rangle\langle k|, \\
\left\langle C^{2}\right\rangle= & \operatorname{Tr}\left[\left\langle\left(U_{I} \rho U_{I}^{\dagger}\right)^{\otimes 2}\right\rangle H_{0}^{\otimes 2}\right] \\
= & \operatorname{Tr}\left[\left\langle\left(G k G^{\dagger} \rho G^{\dagger} K^{\dagger} G\right)^{\otimes 2}\right\rangle H_{0}^{\otimes 2}\right], \\
= & \operatorname{Tr}\left[\left\langle G^{\otimes 4}\left(K \otimes K^{\dagger}\right)^{\otimes 2} G^{\dagger \otimes 4}\right\rangle\right. \\
& \left.\times\left(\rho \otimes H_{0}\right)^{\otimes 2)}\right], \\
\left\langle G^{\otimes 4}\left(K \otimes K^{\dagger}\right)^{\otimes 2} G^{\dagger \otimes 4}\right\rangle= & \sum_{i} \lambda_{i} \Pi_{i}, \\
\lambda_{i}= & \left(\operatorname{Tr} \Pi_{i}\right)^{-1} \operatorname{Tr}\left[\Pi_{i} K^{\dagger 2} \otimes K^{\dagger \otimes 2}\right], \\
\left(\rho \otimes H_{0}\right)^{\otimes 2}= & \sum_{a b c d e f} \rho_{a b} \epsilon_{c} \rho_{d e} \epsilon_{f}|a c d f\rangle\langle b c e f|, \\
\left\langle C^{2}\right\rangle= & \sum_{i} \lambda_{i} \operatorname{Tr}\left[\Pi_{i}\left(\rho \otimes H_{0}\right)^{2}\right] .
\end{aligned}
$$

We then start with the construction of the projectors in a basis, which we take as the computational basis:

$$
\begin{aligned}
& S_{(1234)}+\cdots \propto \sum_{i j k l}|l i j k\rangle\langle i j k l|+| k i l j\rangle\langle i j k l|+| l k i j\rangle\langle i j k l|+| j l i k\rangle\langle i j k l|+| k l j i\rangle\langle i j k l|+| j k l i\rangle\langle i j k l| \\
& S_{(123)}+\cdots \propto \sum_{i j k l}|k i j l\rangle\langle i j k l|+| j k i l\rangle\langle i j k l|+| i l j k\rangle\langle i j k l|+| i k l j\rangle\langle i j k l|+| l j i k\rangle\langle i j k l|+| k j l i\rangle\langle i j k l|+| l i k j\rangle\langle i j k l| \\
& +|j l k i\rangle\langle i j k l|, \\
& S_{(1)} \propto \sum_{i j k l}|i j k l\rangle\langle i j k l|, \\
& S_{(12)}+\cdots \propto \sum_{i j k l}|j i k l\rangle\langle i j k l|+| k j i l\rangle\langle i j k l|+| l j k i\rangle\langle i j k l|+| i k j l\rangle\langle i j k l|+| i l k j\rangle\langle i j k l|+| i j l k\rangle\langle i j k l|, \\
& S_{(12)(34)}+\cdots \propto \sum_{i j k l}|j i l k\rangle\langle i j k l|+| k l i j\rangle\langle i j k l|+| l k j i\rangle\langle i j k l| .
\end{aligned}
$$

Since we are interested only in the scaling with $n$ of the fluctuations, we focus on the structure of the traces and not on the proportionality constants. Using the definitions above, the projectors can then be written explicitly in the computational basis. At this point, we can start the evaluation of the traces. First we note that $\left(\rho \otimes H_{0}\right)^{2}=\sum_{a b c d e f} \rho_{a b} \epsilon_{c} \rho_{d e} \epsilon_{f}|a c d f\rangle\langle b c e f|$. We then have for $\operatorname{Tr}\left[\Pi\left(\rho \otimes H_{0}\right)^{\otimes 2}\right]$ :

$$
\begin{aligned}
\operatorname{Tr}\left[\Pi^{(\operatorname{tr})}\left(\rho \otimes H_{0}\right)^{\otimes 2}\right] \propto & \sum\left[\rho_{a a} \epsilon_{c} \rho_{d d} \epsilon_{f}+\rho_{a d} \epsilon_{a} \rho_{d e} \epsilon_{f}+\rho_{a c} \epsilon_{d} \rho_{d a} \epsilon_{f}+\rho_{a a} \epsilon_{f} \rho_{d c} \epsilon_{d}+\rho_{a a} \epsilon_{d} \rho_{d f} \epsilon_{c}+\rho_{a f} \epsilon_{c} \rho_{d a} \epsilon_{d}\right. \\
& +\rho_{a d} \epsilon_{c} \rho_{d f} \epsilon_{a}+\rho_{a f} \epsilon_{a} \rho_{d d} \epsilon_{c}+\rho_{a c} \epsilon_{f} \rho_{d d} \epsilon_{a}+\rho_{a c} \epsilon_{a} \rho_{d f} \epsilon_{d}+\rho_{a d} \epsilon_{f} \rho_{d a} \epsilon_{c}+\rho_{a f} \epsilon_{d} \rho_{d c} \epsilon_{a} \\
& +\rho_{a d} \epsilon_{a} \rho_{d f} \epsilon_{c}+\rho_{a f} \epsilon_{d} \rho_{d a} \epsilon_{c}+\rho_{a c} \epsilon_{f} \rho_{d a} \epsilon_{d}+\rho_{a d} \epsilon_{f} \rho_{d a} \epsilon_{a}+\rho_{a c} \epsilon_{d} \rho_{d f} \epsilon_{a}+\rho_{a c} \epsilon_{a} \rho_{d d} \epsilon_{f} \\
& \left.+\rho_{a f} \epsilon_{a} \rho_{d c} \epsilon_{d}+\rho_{a d} \epsilon_{e} \rho_{d a} \epsilon_{f}+\rho_{a f} \epsilon_{c} \rho_{d d} \epsilon_{a}+\rho_{a a} \epsilon_{d} \rho_{d c} \epsilon_{f}+\rho_{a a} \epsilon_{f} \rho_{d d} \epsilon_{c}+\rho_{a a} \epsilon_{c} \rho_{d f} \epsilon_{d}\right], \\
\operatorname{Tr}\left[\Pi^{(\mathrm{sig})}\left(\rho \otimes H_{0}\right)^{\otimes 2}\right] \propto & \sum\left[\rho_{a a} \epsilon_{c} \rho_{d d} \epsilon_{f}+\left(\rho_{a d} \epsilon_{a} \rho_{d e} \epsilon_{f}+\rho_{a c} \epsilon_{d} \rho_{d a} \epsilon_{f}+\rho_{a a} \epsilon_{f} \rho_{d c} \epsilon_{d}+\rho_{a a} \epsilon_{d} \rho_{d f} \epsilon_{c}+\rho_{a f} \epsilon_{c} \rho_{d a} \epsilon_{d}\right.\right. \\
& \left.+\rho_{a d} \epsilon_{c} \rho_{d f} \epsilon_{a}+\rho_{a f} \epsilon_{a} \rho_{d d} \epsilon_{c}+\rho_{a c} \epsilon_{f} \rho_{d d} \epsilon_{a}+\rho_{a c} \epsilon_{a} \rho_{d f} \epsilon_{d}+\rho_{a d} \epsilon_{f} \rho_{d a} \epsilon_{c}+\rho_{a f} \epsilon_{d} \rho_{d c} \epsilon_{a}\right) \\
& -\left(\rho_{a f} \epsilon_{a} \rho_{d c} \epsilon_{d}+\rho_{a d} \epsilon_{a} \rho_{d f} \epsilon_{c}+\rho_{a f} \epsilon_{d} \rho_{d a} \epsilon_{c}+\rho_{a c} \epsilon_{f} \rho_{d a} \epsilon_{d}+\rho_{a d} \epsilon_{f} \rho_{d a} \epsilon_{a}+\rho_{a c} \epsilon_{d} \rho_{d f} \epsilon_{a}\right. \\
& \left.\left.+\rho_{a c} \epsilon_{a} \rho_{d d} \epsilon_{f}+\rho_{a d} \epsilon_{e} \rho_{d a} \epsilon_{f}+\rho_{a f} \epsilon_{c} \rho_{d d} \epsilon_{a}\right)+\rho_{a a} \epsilon_{d} \rho_{d c} \epsilon_{f}+\rho_{a a} \epsilon_{f} \rho_{d d} \epsilon_{c}+\rho_{a a} \epsilon_{c} \rho_{d f} \epsilon_{d}\right],
\end{aligned}
$$

$$
\begin{aligned}
\operatorname{Tr}\left[\Pi^{(\mathrm{st})}\left(\rho \otimes H_{0}\right)^{\otimes 2}\right] \propto & \sum\left[\left(\rho_{a c} \epsilon_{a} \rho_{d d} \epsilon_{f}+\rho_{a d} \epsilon_{c} \rho_{d a} \epsilon_{f}+\rho_{a f} \epsilon_{c} \rho_{d d} \epsilon_{a}+\rho_{a a} \epsilon_{d} \rho_{d c} \epsilon_{f}+\rho_{a a} \epsilon_{f} \rho_{d d} \epsilon_{c}+\rho_{a a} \epsilon_{c} \rho_{d f} \epsilon_{d}\right)\right. \\
& -\left(\rho_{a f} \epsilon_{a} \rho_{d c} \epsilon_{d}+\rho_{a d} \epsilon_{a} \rho_{d c} \epsilon_{d}+\rho_{a f} \epsilon_{d} \rho_{d a} \epsilon_{c}+\rho_{a c} \epsilon_{f} \rho_{d a} \epsilon_{d}+\rho_{a d} \epsilon_{f} \rho_{d a} \epsilon_{a}+\rho_{a c} \epsilon_{d} \rho_{d f} \epsilon_{a}\right) \\
& \left.-\left(\rho_{a c} \epsilon_{a} \rho_{d f} \epsilon_{d}+\rho_{a d} \epsilon_{f} \rho_{d a} \epsilon_{c}+\rho_{a f} \epsilon_{d} \rho_{d c} \epsilon_{a}\right)\right],
\end{aligned}
$$




$$
\begin{aligned}
\operatorname{Tr}\left[\Pi^{(s t \otimes s g n)}\left(\rho \otimes H_{0}\right)^{\otimes 2}\right] \propto & \sum\left[-\left(\rho_{a c} \epsilon_{a} \rho_{d d} \epsilon_{f}+\rho_{a d} \epsilon_{c} \rho_{d a} \epsilon_{f}+\rho_{a f} \epsilon_{c} \rho_{d d} \epsilon_{a}+\rho_{a a} \epsilon_{d} \rho_{d c} \epsilon_{f}+\rho_{a a} \epsilon_{f} \rho_{d d} \epsilon_{c}+\rho_{a a} \epsilon_{c} \rho_{d f} \epsilon_{d}\right)\right. \\
& +\left(\rho_{a f} \epsilon_{a} \rho_{d c} \epsilon_{d}+\rho_{a d} \epsilon_{a} \rho_{d c} \epsilon_{d}+\rho_{a f} \epsilon_{d} \rho_{d a} \epsilon_{c}+\rho_{a c} \epsilon_{f} \rho_{d a} \epsilon_{d}+\rho_{a d} \epsilon_{f} \rho_{d a} \epsilon_{a}+\rho_{a c} \epsilon_{d} \rho_{d f} \epsilon_{a}\right) \\
& \left.-\left(\rho_{a c} \epsilon_{a} \rho_{d f} \epsilon_{d}+\rho_{a d} \epsilon_{f} \rho_{d a} \epsilon_{c}+\rho_{a f} \epsilon_{d} \rho_{d c} \epsilon_{a}\right)\right]
\end{aligned}
$$

$$
\begin{aligned}
\operatorname{Tr}\left[\Pi^{(2 \mathrm{D})}\left(\rho \otimes H_{0}\right)^{\otimes 2}\right] \propto & \sum\left[-\left(\rho_{a d} \epsilon_{a} \rho_{d c} \epsilon_{f}+\rho_{a c} \epsilon_{d} \rho_{d a} \epsilon_{f}+\rho_{a a} \epsilon_{f} \rho_{d c} \epsilon_{d}+\rho_{a a} \epsilon_{d} \rho_{d f} \epsilon_{c}+\rho_{a f} \epsilon_{c} \rho_{d a} \epsilon_{d}+\rho_{a f} \epsilon_{a} \rho_{d d} \epsilon_{c}\right.\right. \\
& \left.\left.+\rho_{a c} \epsilon_{f} \rho_{d d} \epsilon_{a}\right)+2\left(\rho_{a c} \epsilon_{a} \rho_{d f} \epsilon_{d}+\rho_{a d} \epsilon_{f} \rho_{d a} \epsilon_{c}+\rho_{a f} \epsilon_{d} \rho_{d c} \epsilon_{a}\right)\right] .
\end{aligned}
$$

We can now evaluate the trace over the operator $K \otimes K^{\dagger}$ with the projectors, $\operatorname{Tr}\left[\Pi K^{\otimes 2} \otimes K^{\dagger \otimes 2}\right]$ 's. We have the following results:

$$
\begin{aligned}
\operatorname{Tr}\left[\Pi^{(\mathrm{tr})} K^{\otimes 2} \otimes K^{\dagger \otimes 2}\right] \propto & \sum_{m n o p} 4 e^{i\left(\theta_{m}+\theta_{p}-\theta_{n}-\theta_{o}\right)}+e^{i\left(\theta_{m}+\theta_{o}-\theta_{m}-\theta_{n}\right)}+e^{i\left(\theta_{o}+\theta_{p}-\theta_{m}-\theta_{n}\right)}+4 e^{i\left(\theta_{p}+\theta_{n}-\theta_{m}-\theta_{o}\right)}+e^{i\left(\theta_{n}+\theta_{o}-\theta_{p}-\theta_{n}\right)} \\
& +4 e^{i\left(\theta_{m}+\theta_{n}-\theta_{o}-\theta_{p}\right)}, \\
& \operatorname{Tr}\left[\Pi^{(\mathrm{sig})} K^{\otimes 2} \otimes K^{\dagger \otimes 2}\right]=0, \\
& \operatorname{Tr}\left[\Pi^{(\mathrm{st})} K^{\otimes 2} \otimes K^{\dagger \otimes 2}\right] \propto \sum_{m n o p}\left[e^{i\left(\theta_{m}+\theta_{n}-\theta_{o}-\theta_{p}\right)}-e^{i\left(\theta_{o}+\theta_{p}-\theta_{m}-\theta_{n}\right)}\right], \\
\operatorname{Tr}\left[\Pi^{(2 \mathrm{D})} K^{\otimes 2} \otimes K^{\dagger \otimes 2}\right] \propto & \sum_{m n o p}\left\{e^{i\left(\theta_{n}+\theta_{o}-\theta_{m}-\theta_{p}\right)}+e^{i\left(\theta_{n}+\theta_{m}-\theta_{p}-\theta_{o}\right)}+2 e^{i\left(\theta_{0}+\theta_{p}-\theta_{m}-\theta_{n}\right)}+2 e^{i\left(\theta_{p}+\theta_{o}-\theta_{n}-\theta_{m}\right)}\right. \\
& \left.-\left[e^{i\left(\theta_{m}+\theta_{p}-\theta_{n}-\theta_{o}\right)}+e^{i\left(\theta_{m}+\theta_{o}-\theta_{p}-\theta_{n}\right)}+e^{i\left(\theta_{o}+\theta_{n}-\theta_{p}-\theta_{m}\right)}+e^{i\left(\theta_{p}+\theta_{m}-\theta_{o}-\theta_{n}\right)}+e^{i\left(\theta_{n}+\theta_{p}-\theta_{o}-\theta_{m}\right)}\right]\right\} .
\end{aligned}
$$

We now consider the traces of the projectors alone, $\operatorname{Tr}[\Pi]$ 's. It is not hard to see that for large values of $n$, we have

$$
\begin{gathered}
\operatorname{Tr}\left[\Pi^{(\mathrm{tr})}\right] \propto n^{4}, \\
\operatorname{Tr}\left[\Pi^{(\mathrm{sig})}\right] \propto n^{4}, \\
\operatorname{Tr}\left[\Pi^{(\mathrm{st})}\right] \propto n^{4}, \\
\operatorname{Tr}\left[\Pi^{(s t \otimes s g n)}\right] \propto n^{4}, \\
\operatorname{Tr}\left[\Pi^{(2 \mathrm{D})}\right] \propto n^{4} .
\end{gathered}
$$

At this point we can calculate the average fluctuations, which can be written as

$$
\begin{gathered}
F=F_{\Pi^{(\mathrm{tr})}}+F_{\Pi^{(\mathrm{sig})}}+F_{\Pi^{(\mathrm{st})}}+F_{\Pi^{(\mathrm{st} \otimes \mathrm{sgn})}}+F_{\Pi^{(2 \mathrm{D})}} \\
F_{\Pi^{(\mathrm{rr})} \propto} \propto \frac{1}{n^{4}}\left[\sum_{m n o p} 4 e^{i\left(\theta_{m}+\theta_{p}-\theta_{n}-\theta_{o}\right)}+4 e^{i\left(\theta_{m}+\theta_{o}-\theta_{m}-\theta_{n}\right)}+4 e^{i\left(\theta_{o}+\theta_{p}-\theta_{m}-\theta_{n}\right)}\right. \\
\left.+4 e^{i\left(\theta_{p}+\theta_{n}-\theta_{m}-\theta_{o}\right)}+4 e^{i\left(\theta_{n}+\theta_{o}-\theta_{p}-\theta_{n}\right)}+4 e^{i\left(\theta_{m}+\theta_{n}-\theta_{o}-\theta_{p}\right)}\right] \\
+\sum\left[\rho_{a a} \epsilon_{c} \rho_{d d} \epsilon_{f}+\rho_{a d} \epsilon_{a} \rho_{d e} \epsilon_{f}+\rho_{a c} \epsilon_{d} \rho_{d a} \epsilon_{f}+\rho_{a a} \epsilon_{f} \rho_{d c} \epsilon_{d}+\rho_{a a} \epsilon_{d} \rho_{d f} \epsilon_{c}+\rho_{a f} \epsilon_{c} \rho_{d a} \epsilon_{d}+\rho_{a d} \epsilon_{c} \rho_{d f} \epsilon_{a}\right. \\
+\rho_{a f} \epsilon_{a} \rho_{d d} \epsilon_{c}+\rho_{a c} \epsilon_{f} \rho_{d d} \epsilon_{a}+\rho_{a c} \epsilon_{a} \rho_{d f} \epsilon_{d}+\rho_{a d} \epsilon_{f} \rho_{d a} \epsilon_{c}+\rho_{a f} \epsilon_{d} \rho_{d c} \epsilon_{a}+\rho_{a f} \epsilon_{a} \rho_{d c} \epsilon_{d}+\rho_{a d} \epsilon_{a} \rho_{d f} \epsilon_{c} \\
+\rho_{a f} \epsilon_{d} \rho_{d a} \epsilon_{c}+\rho_{a c} \epsilon_{f} \rho_{d a} \epsilon_{d}+\rho_{a d} \epsilon_{f} \rho_{d a} \epsilon_{a}+\rho_{a c} \epsilon_{d} \rho_{d f} \epsilon_{a}+\rho_{a c} \epsilon_{a} \rho_{d d} \epsilon_{f}+\rho_{a d} \epsilon_{e} \rho_{d a} \epsilon_{f}+\rho_{a f} \epsilon_{c} \rho_{d d} \epsilon_{a} \\
\left.+\rho_{a a} \epsilon_{d} \rho_{d c} \epsilon_{f}+\rho_{a a} \epsilon_{f} \rho_{d d} \epsilon_{c}+\rho_{a a} \epsilon_{c} \rho_{d f} \epsilon_{d}\right], \\
F_{\Pi^{(\mathrm{st})}} \propto \frac{1}{n^{4}}\left[\sum_{m n o p} 4 e^{i\left(\theta_{m}+\theta_{n}-\theta_{o}-\theta_{p}\right)}-4 e^{i\left(\theta_{o}+\theta_{p}-\theta_{m}-\theta_{n}\right)}\right]
\end{gathered}
$$




$$
\begin{aligned}
& \left\{3 \operatorname{Tr}\left[\left(\rho \otimes H_{0}\right)^{\otimes 2}\right]-\sum\left[\left(\rho_{a c} \epsilon_{a} \rho_{d d} \epsilon_{f}+\rho_{a d} \epsilon_{c} \rho_{d a} \epsilon_{f}+\rho_{a f} \epsilon_{c} \rho_{d d} \epsilon_{a}+\rho_{a a} \epsilon_{d} \rho_{d c} \epsilon_{f}+\rho_{a a} \epsilon_{f} \rho_{d d} \epsilon_{c}+\rho_{a a} \epsilon_{c} \rho_{d f} \epsilon_{d}\right)\right.\right. \\
& -\left(\rho_{a f} \epsilon_{a} \rho_{d c} \epsilon_{d}+\rho_{a d} \epsilon_{a} \rho_{d c} \epsilon_{d}+\rho_{a f} \epsilon_{d} \rho_{d a} \epsilon_{c}+\rho_{a c} \epsilon_{f} \rho_{d a} \epsilon_{d}+\rho_{a d} \epsilon_{f} \rho_{d a} \epsilon_{a}+\rho_{a c} \epsilon_{d} \rho_{d f} \epsilon_{a}\right) \\
& \left.\left.-\left(\rho_{a c} \epsilon_{a} \rho_{d f} \epsilon_{d}+\rho_{a d} \epsilon_{f} \rho_{d a} \epsilon_{c}+\rho_{a f} \epsilon_{d} \rho_{d c} \epsilon_{a}\right)\right]\right\} \\
& F_{\Pi^{(s t \otimes s g n)}} \propto \frac{1}{n^{4}}\left[\sum_{\text {mnop }} e^{i\left(\theta_{o}+\theta_{p}-\theta_{m}-\theta_{n}\right)}\right] \cdot\left\{\frac{3}{24} 3 \operatorname{Tr}\left[\left(\rho \otimes H_{0}\right)^{\otimes 2}\right]+\left[\sum-\left(\rho_{a c} \epsilon_{a} \rho_{d d} \epsilon_{f}+\rho_{a d} \epsilon_{c} \rho_{d a} \epsilon_{f}+\rho_{a f} \epsilon_{c} \rho_{d d} \epsilon_{a}\right.\right.\right. \\
& \left.+\rho_{a a} \epsilon_{d} \rho_{d c} \epsilon_{f}+\rho_{a a} \epsilon_{f} \rho_{d d} \epsilon_{c}+\rho_{a a} \epsilon_{c} \rho_{d f} \epsilon_{d}\right)+\left(\rho_{a f} \epsilon_{a} \rho_{d c} \epsilon_{d}+\rho_{a d} \epsilon_{a} \rho_{d c} \epsilon_{d}+\rho_{a f} \epsilon_{d} \rho_{d a} \epsilon_{c}+\rho_{a c} \epsilon_{f} \rho_{d a} \epsilon_{d}\right. \\
& \left.\left.\left.+\rho_{a d} \epsilon_{f} \rho_{d a} \epsilon_{a}+\rho_{a c} \epsilon_{d} \rho_{d f} \epsilon_{a}\right)-\left(\rho_{a c} \epsilon_{a} \rho_{d f} \epsilon_{d}+\rho_{a d} \epsilon_{f} \rho_{d a} \epsilon_{c}+\rho_{a f} \epsilon_{d} \rho_{d c} \epsilon_{a}\right)\right]\right\} \text {, } \\
& F_{\Pi^{(2 \mathrm{D})}} \propto \frac{1}{n^{4}} \sum_{m n o p}\left[e^{i\left(\theta_{n}+\theta_{o}-\theta_{m}-\theta_{p}\right)}-\left(e^{i\left(\theta_{m}+\theta_{p}-\theta_{n}-\theta_{o}\right)}+e^{i\left(\theta_{m}+\theta_{o}-\theta_{p}-\theta_{n}\right)}+e^{i\left(\theta_{o}+\theta_{n}-\theta_{p}-\theta_{m}\right)}\right.\right. \\
& \left.\left.+e^{i\left(\theta_{p}+\theta_{m}-\theta_{o}-\theta_{n}\right)}+e^{i\left(\theta_{n}+\theta_{p}-\theta_{o}-\theta_{m}\right)}\right)+2 e^{i\left(\theta_{n}+\theta_{m}-\theta_{p}-\theta_{o}\right)}+2 e^{i\left(\theta_{0}+\theta_{p}-\theta_{m}-\theta_{n}\right)}+2 e^{i\left(\theta_{p}+\theta_{o}-\theta_{n}-\theta_{m}\right)}\right] \\
& \cdot\left\{2 \operatorname{Tr}\left[\left(\rho \otimes H_{0}\right)^{\otimes 2}\right]+\sum-\left(\rho_{a d} \epsilon_{a} \rho_{d c} \epsilon_{f}+\rho_{a c} \epsilon_{d} \rho_{d a} \epsilon_{f}\right.\right. \\
& +\rho_{a a} \epsilon_{f} \rho_{d c} \epsilon_{d}+\rho_{a a} \epsilon_{d} \rho_{d f} \epsilon_{c}+\rho_{a f} \epsilon_{c} \rho_{d a} \epsilon_{d}+\rho_{a f} \epsilon_{a} \rho_{d d} \epsilon_{c} \\
& \left.\left.+\rho_{a c} \epsilon_{f} \rho_{d d} \epsilon_{a}\right)+2\left(\rho_{a c} \epsilon_{a} \rho_{d f} \epsilon_{d}+\rho_{a d} \epsilon_{f} \rho_{d a} \epsilon_{c}+\rho_{a f} \epsilon_{d} \rho_{d c} \epsilon_{a}\right)\right\} \text {. }
\end{aligned}
$$

\section{Concentration bound}

Let us now consider an upper bound for the nonzero fluctuation terms based on general grounds and on the von Neumann inequality [67]. Let $A$ and $B$ be Hermitean matrices with eigenvalues values of $a_{i} \geqslant a_{i-1}$ 's and $b_{i} \geqslant b_{i-1}$. Then, we have

$$
|\operatorname{Tr}(A B)| \leqslant \sum_{i=1}^{n} a_{i} b_{i} .
$$

Let us now assume that $A$ is a projector with $k$ nonzero eigenvalues. Then the inequality implies that

$$
|\operatorname{Tr}(\Pi B)| \leqslant \sum_{j=n-k}^{n} b_{j},
$$

where $b_{n} \cdots b_{n-k}$ are the highest $k$ 's eigenvalues values of $B$. We thus need to focus on the singular values of $(\rho \otimes H)^{\otimes 2}$. The eigenvalues of $\rho \otimes H$, are $e_{i j}=p_{i} \epsilon_{j}$, and the eigenvalues $(\rho \otimes H)^{\otimes 2}$ are $e_{i j k l}=p_{i} \epsilon_{j} p_{k} \epsilon_{l}$. Since $p_{i} \leqslant 1$ in the most general case, $e_{i j k l}$ is upper bounded by $\epsilon_{\max }^{2}$. We thus have that a conservative upper bound is given by

$$
\left|\operatorname{Tr}\left(\Pi(\rho \otimes H)^{\otimes 2}\right)\right| \leqslant k^{2} \epsilon_{\max }^{2},
$$

where $k$ is the dimension of the nonzero subspace of the projector operator. Since each term of the trace is divided by the dimension of the projector operator and we have four nonzero terms, we have

$$
F \leqslant 4 \epsilon_{\max }^{2}
$$

in the most general case. However, the bound $p_{i} \leqslant 1$ is very loose. If $p_{i} \leqslant \frac{\gamma}{n}$, then we have

$$
F \leqslant 4 \gamma^{2} \frac{\epsilon_{\mathrm{max}}^{2}}{n^{2}}
$$

and thus there is concentration. For instance, we have concentration if we have that $\rho$ is a mixed sate. A stronger bound can be done by using the expressions we derived. We see from the bound above that this is not enough to prove concentration. However, the concentration can be proven if the take advantage of the structure of the fluctuations in terms of the density matrix.

We now provide an alternative proof of the same statement. From the previous subsection, we see that the work fluctuations $F$ can be upper bounded as

$$
F \leqslant C n^{-4} M(n) k n^{2},
$$

where $C$ is a $O(1)$ constant counting the number of all the terms in $F, M(n)$ is an upper bound over al the terms of the type $\left|\sum_{\text {mnop }} e^{i\left(\theta_{o}+\theta_{p}-\theta_{m}-\theta_{n}\right)}\right|$, and $k n^{2}$ is the upper bound to the terms containing the $\rho, H_{0}$ :

$$
\begin{aligned}
\left|\operatorname{Tr}\left[\Pi_{x}\left(\rho \otimes H_{0}\right)^{\otimes 2}\right]\right| & \leqslant\left|\operatorname{Tr}\left[\left(\rho \otimes H_{0}\right)^{\otimes 2}\right]\right| \\
& =(\operatorname{Tr} \rho)^{2}\left(\operatorname{Tr} H_{0}\right)^{2} \\
& =\left(\operatorname{Tr} H_{0}\right)^{2} \leqslant k n^{2},
\end{aligned}
$$

which is true because the projector operators are positive. Putting things together, we obtain

$$
F \leqslant C^{\prime} M(n) n^{-2}
$$

with a new constant $C^{\prime}$. The fluctuations are thus ruled by $M(n)$. One can design quantum batteries with large fluctuations. However, on average these fluctuations go to zero. Indeed, it should not be surprising that the sum over (the sum of) random phases goes to zero. For random unitaries we need to use the ensemble of CUE. Numerical evaluation (see in Fig. 4) shows that $M(n)$ is concentrated around zero for large dimension $n$. 

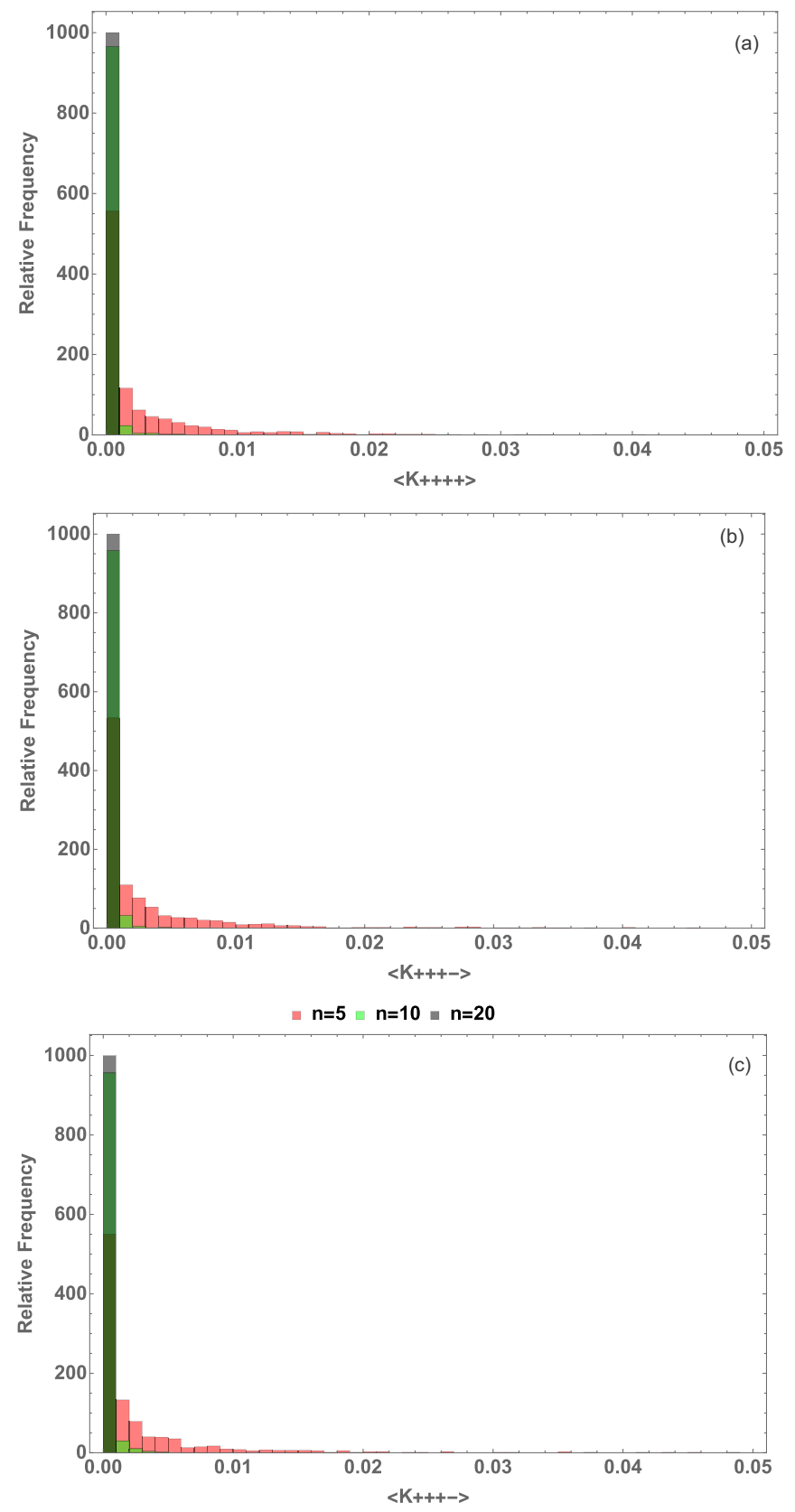

FIG. 4. Frequency distribution of the average $\left\langle e^{\sum_{j=1}^{4} \pm i \theta_{j}}\right\rangle$ for CUE over $M=1000$ samples. We see that the distribution is strongly peaked around the value of $\left\langle K^{2}\right\rangle=0$, for the three terms with three possible signatures in the exponent for (a)-(c), which is what is necessary for the proof of our concentration at least in the case of CUE. The last peak is just a binning artifact.

\section{Jaynes-Cummings model}

As seen in Eq. (12), the average work depends only on the value of the eigenvalues of the unitary evolution operator $K$. Let us consider the case of an optical cavity interacting with a two-state system. The optical cavity with the two-state system (an atom) $\operatorname{span}(|g\rangle,|e\rangle)$ can be described within the rotatingwave approximation using the Jaynes-Cumming Hamiltonian:

$$
H=\omega a^{\dagger} a+\frac{\Omega}{2} \sigma_{z}+g(t)\left(a \sigma_{+}+a^{\dagger} \sigma_{-}\right) \equiv H_{0}+V(t) .
$$

It is immediate to see that $\left[H, a^{+} a+\sigma_{z}\right]=0$. Specifically, we focus on the interaction picture, in which $H_{I}=R H R^{\dagger}$, where (in the rotating frame) we have $R=e^{-i \omega t\left(a^{\dagger} a+\frac{\sigma_{z}}{2}\right)}$, and one has a Hamiltonian described by $H_{I}=R H R^{\dagger}$, with

$$
H_{I}=\frac{\Omega-\omega}{2} \sigma_{z}+g(t)\left(a^{\dagger} \sigma_{-}+\sigma_{+} a\right)
$$

We define $\Delta=\Omega-\omega$. The operators $a$ and $a^{\dagger}$ act on the electromagnetic field, while $\sigma$ 's act on the two-level system. We have

$$
\sigma_{+}=|e\rangle\left\langle g\left|, \quad \sigma_{-}=\right| g\right\rangle\langle e| .
$$

We now consider a wave function of the form

$$
|\psi(t)\rangle=\sum_{n=0}^{R} C_{n}(t)|n\rangle \otimes|e\rangle+D_{n}(t)|n+1\rangle \otimes|g\rangle,
$$

where we will send $R \rightarrow \infty$ at the end of the calculation. The time evolution of this system is given by the Schroedinger equation (in the interaction picture), which is of the form:

$$
i \partial_{t}|\psi(t)\rangle=H_{I}|\psi(t)\rangle,
$$

which is not hard to see that it can be written as

$$
\begin{aligned}
i \partial_{t}\left[\begin{array}{l}
C_{n}(t) \\
D_{n}(t)
\end{array}\right] & =V\left[\begin{array}{l}
C_{n}(t) \\
D_{n}(t)
\end{array}\right] \\
& =\left(\begin{array}{cc}
\frac{\Delta}{2} & \sqrt{n+1} g \\
\sqrt{n+1} g & -\frac{\Delta}{2}
\end{array}\right)\left[\begin{array}{c}
C_{n}(t) \\
D_{n}(t)
\end{array}\right],
\end{aligned}
$$

whose solution is given by

$$
\left|\psi_{n}(t)\right\rangle=\mathcal{T} e^{-i \int^{t} V\left(t^{\prime}\right) d t^{\prime}}\left|\psi_{n}(0)\right\rangle .
$$

We note that $V\left(t^{\prime}\right) V(t) \neq V(t) V\left(t^{\prime}\right)$ in the case of a timedependent interaction Hamiltonian. In fact, we see that on the $n$th subspace of the wave function, given the definition $W(\Delta, g)=\Delta g^{\prime}-g \Delta^{\prime}$ of the Wronskian of the functions $\Delta$ and $g$, we have

$$
\left[V_{r}\left(t^{\prime}\right), V_{r}(t)\right]=\left[\begin{array}{cc}
0 & W(\Delta, g) \sqrt{r+1} \\
W(\Delta, g) \sqrt{r+1} & 0
\end{array}\right]
$$

from which we observe that we can have a time-dependent and commuting (at all times) Hamiltonian if we have the condition

$$
\Delta g^{\prime}=g \Delta^{\prime}
$$

which can be satisfied if

$$
\frac{\Delta(t)}{\Delta\left(t^{\prime}\right)}=\frac{g(t)}{g\left(t^{\prime}\right)}=e^{M\left(t-t^{\prime}\right)}
$$

for a constant $M$. In this case, the time ordering can be removed and we can write

$$
\int_{t_{0}}^{t} V_{r}\left(t^{\prime}\right) d t^{\prime}=\left(\begin{array}{cc}
\frac{\Delta}{2} & \sqrt{r+1} g \\
\sqrt{r+1} g & -\frac{\Delta}{2}
\end{array}\right) \frac{e^{M t}-e^{M t_{0}}}{M} .
$$

The Stone operator in this case can also be written explicitly on each subspace. It can be shown that in each $r$ th 
subspaces

$$
e^{-i \int_{t_{0}}^{t} V_{r}\left(t^{\prime}\right) d t^{\prime}}=\mathbb{I} \sum_{k=0}^{\infty} \frac{(-1)^{k} \beta_{r}(t)^{2 k}}{(2 k) !}-i \frac{\hat{\sigma}_{x}}{\beta_{r}(t)} \sum_{k=0}^{\infty}(-1)^{k} \frac{\beta_{r}(t)^{2 k+1}}{(2 k+1) !}
$$

and where

$$
\beta_{r}(t)=\left[\frac{\Delta_{0}^{2}}{4}+g^{2}(r+1)\right]\left(\frac{e^{M t}-e^{M t_{0}}}{M}\right)^{2} .
$$

Thus, the Stone operator which describes the time evolution on the $r$ th subspace is given by

$$
e^{-i \int_{t_{0}}^{t} V_{r}\left(t^{\prime}\right) d t^{\prime}}=\left\{\begin{array}{cc}
\cos \left[\beta_{r}(t)\right]-\frac{i \Delta}{2 \beta_{r}(t)} \sin \left[\beta_{r}(t)\right] & -i \frac{g \sqrt{r+1}}{\beta_{r}(t)} \sin \left[\beta_{r}(t)\right] \\
-i \frac{g \sqrt{r+1}}{\beta_{r}(t)} \sin \left[\beta_{r}(t)\right] & \cos \left[\beta_{r}(t)\right]+\frac{i \Delta}{2 \beta_{r}(t)} \sin \left[\beta_{r}(t)\right]
\end{array}\right\} .
$$

We now focus on the eigenvalues of the matrix above, which must be of the form $e^{i \theta_{k}}$. For a matrix of the type

$$
\left(\begin{array}{cc}
a-i d & -i c \\
-i c & a+i d
\end{array}\right)
$$

the eigenvalues are known exactly and are of the form $\lambda_{ \pm}=a \pm i \sqrt{c^{2}+d^{2}}$. It is immediate to see that the eigenvalues are complex and have norm 1 . The phases are given by $\pm \theta_{k} \equiv \pm \beta_{k}(t)$. We thus find that

$$
\theta_{k}-\theta_{m}=g_{0}^{2}(k-m)\left(\frac{e^{M t}-e^{M t_{0}}}{M^{2}}\right)^{2}
$$

which is what we need for the evaluation for the work in the main text. We can now plug this result into Eq. (12), which reads

$$
\langle W(t)\rangle_{V}=E_{0}\left[1-\frac{2 \sum_{j \neq k} \cos \left(\theta_{j}-\theta_{k}\right)+1-n}{n^{2}-1}\right]-\frac{n^{2}-\left(2 \sum_{j \neq k} \cos \left(\theta_{j}-\theta_{k}\right)+n\right)}{n^{2}-1} \frac{\operatorname{Tr}\left(H_{0}\right)}{n},
$$

where here $n=2 R$, and $R$ is the number of modes of the electric field. Let us call $\alpha=g_{0}^{2}\left(\frac{e^{M t}-e^{M_{0}}}{M^{2}}\right)^{2}$. We thus need to calculate $\sum_{j \neq k} \cos [\alpha(j-k)]$. Thankfully, this sum is known and is given by

$$
\begin{aligned}
\tilde{Q}(\alpha) \equiv & \sum_{i=1}^{n} \sum_{j=i+1}^{n} \cos [\alpha(i-j)] \\
= & \frac{1}{4}\left\{\cos \left(\frac{\alpha-\pi}{2}\right) \csc \left(\frac{\alpha}{2}\right)-\sin \left(\frac{\alpha-\pi}{2}\right) \cot \left(\frac{\alpha}{2}\right) \csc \left(\frac{\alpha}{2}\right)-\csc \left(\frac{\alpha}{2}\right)\right. \\
& \left.\times \cos \left[\frac{1}{2}(\alpha-2 \alpha n-\pi)\right]+\cot \left(\frac{\alpha}{2}\right) \csc \left(\frac{\alpha}{2}\right) \sin \left[\frac{1}{2}(\alpha-2 \alpha n-\pi)\right]-2 n\right\},
\end{aligned}
$$

from which we obtain:

$$
\langle W(t)\rangle_{V}=E_{0}\left[1-\frac{4 \tilde{Q}\left(\alpha_{t}\right)+1-n}{n^{2}-1}\right]-\frac{n^{2}-\left[4 \tilde{Q}\left(\alpha_{t}\right)+n\right]}{n^{2}-1} \frac{\operatorname{Tr}\left(H_{0}\right)}{n}=W_{0}-\tilde{Q}\left(\alpha_{t}\right) W_{1},
$$

with

$$
\begin{aligned}
& W_{0}=E_{0}\left(1+\frac{1}{n+1}\right)-\frac{\operatorname{Tr}\left(H_{0}\right)}{n+1} \\
& W_{1}=\frac{4}{n^{2}-1}\left[\frac{\operatorname{Tr}\left(H_{0}\right)}{n}-E_{0}\right] .
\end{aligned}
$$

We thus see that the time dependence of the work enters only in $\tilde{Q}[\alpha(t)]$.

In order to calculate the times at which the revivals occur, we write $\tilde{Q}(\alpha)$ in terms of $\alpha(t)=2 \pi z(t)$. We thus have a simpler formula:

$$
\tilde{Q}[r(t)]=\frac{1}{4} \csc ^{2}[\pi z(t)]\{n \cos [2 \pi z(t)]-\cos [2 \pi n z(t)]-n+1\} .
$$

It is not hard to see that revivals occur for $z_{k}=k$ with $k \in \mathbb{N}$ and thus for $\alpha_{k}$ a multiple of $2 \pi$. We now have that

$$
\alpha_{k}=g_{0}^{2}\left(\frac{e^{M t_{k}}-e^{M t_{0}}}{M^{2}}\right)^{2}=2 \pi k
$$


for $k \in \mathbb{N}$, from which we get the revival times

$$
t_{k}=\frac{\log \left(\frac{g_{0} e^{t_{0} M}+\sqrt{2 \pi} M \sqrt{k}}{g_{0}}\right)}{M}
$$

as a function of $M$ and $g_{0}$.

\section{Time-dependent perturbation theory}

In the case of the Jaynes-Cummings model we could solve for the time evolution exactly. This is rarely the case and we must resort to perturbation theory in most cases. Consider to start the definition of the work:

$$
W=\operatorname{Tr}\left(\rho_{0} H_{0}\right)-\operatorname{Tr}\left(U_{I} \rho_{0} U_{I}^{\dagger} H_{0}\right),
$$

where we consider a Dyson expansion. In this case, the solution is given by the Dyson time ordering

$$
U_{I}(t)=\mathcal{T} \sum_{k=0}^{\infty} \frac{(-i)^{k}}{k !}\left[\int_{0}^{t} d t^{\prime} V_{I}\left(t^{\prime}\right)\right]^{k} .
$$

We are interested in the case in which we need to resort to perturbation theory to evaluate the unitary operator above. To the second order, we have

$$
\begin{aligned}
& U_{2}=G^{\dagger}\left[\mathbb{I}-i \int_{t_{0}}^{t} V_{0}\left(t^{\prime}\right) d t^{\prime}-\frac{1}{2} \int_{t_{0}}^{t} \int_{t_{0}}^{t}: V_{0}\left(t^{\prime}\right) V_{0}\left(t^{\prime \prime}\right): d t^{\prime} d t^{\prime \prime}\right] G+O\left(t^{3}\right), \\
& U_{2}^{\dagger}=G^{\dagger}\left\{\mathbb{I}+i \int_{t_{0}}^{t} V_{0}^{\dagger}\left(t^{\prime}\right) d t^{\prime}-\frac{1}{2} \int_{t_{0}}^{t} \int_{t_{0}}^{t}\left[: V_{0}\left(t^{\prime}\right) V_{0}\left(t^{\prime \prime}\right):\right]^{\dagger} d t^{\prime} d t^{\prime \prime}\right\} G+O\left(t^{3}\right) .
\end{aligned}
$$

In what follows, we can assume that $V_{0}^{\dagger}=V_{0}$. Given the expressions above, we have now to evaluate the average of

$$
W=\operatorname{Tr}\left(\rho_{0} H_{0}\right)-\operatorname{Tr}\left(G^{\dagger} U_{2} G \rho_{0} G^{\dagger} U_{2}^{\dagger} G H_{0}\right)
$$

using the average of the unitary matrix $G$ :

$$
\left\langle\left(G^{\dagger} \otimes G^{\dagger}\right)\left(U_{2} \otimes U_{2}^{\dagger}\right)(G \otimes G)\right\rangle_{G}=\lambda_{+} \Pi_{+}+\lambda_{-} \Pi_{-},
$$

with

$$
\begin{aligned}
& \lambda_{+}=\frac{\operatorname{Tr}\left[\left(U_{2} \otimes U_{2}^{\dagger}\right) \Pi_{+}\right]}{\operatorname{Tr}\left(\Pi_{+}\right)}=\frac{2}{n(n+1)} \frac{\operatorname{Tr}\left(U_{2}\right) \operatorname{Tr}\left(U_{2}^{\dagger}\right)+\operatorname{Tr}\left(U_{2} U_{2}^{\dagger}\right)}{2} \\
& \lambda_{-}=\frac{\operatorname{Tr}\left[\left(U_{2} \otimes U_{2}^{\dagger}\right) \Pi_{-}\right]}{\operatorname{Tr}\left(\Pi_{-}\right)}=\frac{2}{n(n-1)} \frac{\operatorname{Tr}\left(U_{2}\right) \operatorname{Tr}\left(U_{2}^{\dagger}\right)-\operatorname{Tr}\left(U_{2} U_{2}^{\dagger}\right)}{2} .
\end{aligned}
$$

Note that $\operatorname{Tr}\left(U_{2} U_{2}^{\dagger}\right)=\operatorname{Tr}\left(U_{2}^{\dagger} U_{2}\right)=n+O\left(t^{3}\right)$. We can use at this point the Eqs. (A12) again. After a rapid calculation we see that (up to corrections of order $t^{3}$ ), we have

$$
\lambda_{ \pm}=\frac{n^{2} \pm n-n \operatorname{Tr}\left(A^{2}\right)-\operatorname{Tr}(A)^{2}}{n^{2} \pm n}
$$

and thus

$$
\begin{aligned}
& \frac{\lambda_{+}+\lambda_{-}}{2}=\frac{n^{2}-1+\operatorname{Tr}(A)^{2}-n \operatorname{Tr}\left(A^{2}\right)}{n^{2}-1} \\
& \frac{\lambda_{+}-\lambda_{-}}{2}=-\frac{\operatorname{Tr}(A)^{2}-n \operatorname{Tr}\left(A^{2}\right)}{n\left(n^{2}-n\right)},
\end{aligned}
$$

where $A=\int_{t_{0}}^{t} V_{0}\left(t^{\prime}\right) d t^{\prime}$, where we used the fact that inside the traces one has $\operatorname{Tr}\left\{\int_{t_{0}}^{t} \int_{t_{0}}^{t}\left[: V_{0}\left(t^{\prime}\right) V_{0}\left(t^{\prime \prime}\right):\right]^{\dagger} d t^{\prime} d t^{\prime \prime}\right\}=\operatorname{Tr}\left[\int_{t_{0}}^{t} \int_{t_{0}}^{t}\right.$ : $\left.V_{0}\left(t^{\prime}\right) V_{0}\left(t^{\prime \prime}\right): d t^{\prime} d t^{\prime \prime}\right]$. We can now write

$$
\begin{aligned}
\langle W\rangle_{G} & =\operatorname{Tr}\left(\rho_{0} H_{0}\right)\left[1-\frac{\lambda_{+}+\lambda_{-}}{2}\right]-\frac{\lambda_{+}-\lambda_{-}}{2} \operatorname{Tr}\left(H_{0}\right) \operatorname{Tr}\left(\rho_{0}\right) \\
& =\operatorname{Tr}\left(\rho_{0} H_{0}\right)\left\{1-\frac{\left[\operatorname{Tr}(A)^{2}-n \operatorname{Tr}\left(A^{2}\right)+n^{2}-1\right]}{n^{2}-1}\right\}+\frac{\operatorname{Tr}(A)^{2}-n \operatorname{Tr}\left(A^{2}\right)}{n^{2}-1} \frac{\operatorname{Tr}\left(H_{0}\right)}{n} . \\
& =\frac{\operatorname{Tr}(A)^{2}-n \operatorname{Tr}\left(A^{2}\right)}{n^{2}-1} \frac{\operatorname{Tr}\left(H_{0}\right)}{n}-\operatorname{Tr}\left(\rho_{0} H_{0}\right) \frac{\left[\operatorname{Tr}(A)^{2}-n \operatorname{Tr}\left(A^{2}\right)\right]}{n^{2}-1} \\
& =\frac{\operatorname{Tr}(A)^{2}-n \operatorname{Tr}\left(A^{2}\right)}{n^{2}-1}\left[\frac{\operatorname{Tr}\left(H_{0}\right)}{n}-\operatorname{Tr}\left(\rho_{0} H_{0}\right)\right] \\
& =\left\langle\Delta A^{2}\right\rangle_{G}\langle E\rangle_{G} .
\end{aligned}
$$


As it could be seen from the beginning, we see again explicitly that the average work is the product of two terms, the first is adimensional and due to the perturbation,

$$
\left\langle\Delta A^{2}\right\rangle_{G}=\frac{\operatorname{Tr}(A)^{2}-n \operatorname{Tr}\left(A^{2}\right)}{n^{2}-1}
$$

and the second term has the dimensions of energy and due to the density matrix only:

$$
\langle E\rangle_{G}=\left[\frac{\operatorname{Tr}\left(H_{0}\right)}{n}-\operatorname{Tr}\left(\rho_{0} H_{0}\right)\right] .
$$

This shows that no work can extracted if the density matrix is the one of a completely mixed state.

\section{a. Example: Harmonic perturbations}

Let us now consider the example of an $n$-level system. At time $t=0$, the system is described by the eigenvalue equation

$$
\hat{H}_{0} \psi_{m}=E_{m} \psi_{m},
$$

and thus the wave function as a function of time can be written as

$$
\psi(t)=\sum_{m} c_{m} e^{-i E_{m} t} \psi_{m}
$$

We consider now a harmonic perturbation of the form:

$$
\hat{H}_{1}(t)=\hat{V} e^{i \omega t}+\hat{V}^{\dagger} e^{-i \omega t},
$$

where $\hat{V}$ is a generic operator and $\hat{V}^{\dagger}$ its Hermitean conjugate.

Then, according to the formulas we have derived, the average work, if we consider random rotations with respect to $G$ of $\hat{H}_{1}(t) \rightarrow G^{\dagger} \hat{H}_{1}(t) G$, depends on

$$
\begin{aligned}
A= & \int_{t_{0}}^{t} d t^{\prime}\left(\hat{V} e^{i \omega t^{\prime}}+\hat{V}^{\dagger} e^{-i \omega t^{\prime}}\right) i \hat{V} \frac{\left(e^{i t_{0} \omega}-e^{i t \omega}\right)}{\omega} \\
& -i \hat{V}^{\dagger} \frac{\left(e^{-i t_{0} \omega}-e^{-i t \omega}\right)}{\omega} \\
A^{2}= & -\hat{V}^{2} \frac{\left(e^{i t_{0} \omega}-e^{i t \omega}\right)^{2}}{\omega^{2}}-\left(\hat{V}^{\dagger}\right)^{2} \frac{\left(e^{-i t_{0} \omega}-e^{-i t \omega}\right)^{2}}{\omega^{2}} \\
& +\left(\hat{V} \hat{V}^{\dagger}+\hat{V}^{\dagger} \hat{V}\right) \frac{\left(e^{-i t_{0} \omega}-e^{-i t \omega}\right)\left(e^{i t_{0} \omega}-e^{i t \omega}\right)}{\omega^{2}} .
\end{aligned}
$$

We now use:

$$
\begin{gathered}
\left(e^{-i t_{0} \omega}-e^{-i t \omega}\right)=-2 i e^{-i \frac{t+t_{0}}{2} \omega} \sin \left(\frac{t-t_{0}}{2} \omega\right) \\
\left(e^{i t_{0} \omega}-e^{i t \omega}\right)=2 i e^{i \frac{t+t_{0}}{2} \omega} \sin \left(\frac{t-t_{0}}{2} \omega\right)
\end{gathered}
$$

and thus, if we define $f(t, \omega)=2 \frac{\sin \left(\frac{t-t_{0}}{2} \omega\right)}{\omega}$, then we have

$$
\begin{aligned}
A & =-f(t, \omega)\left(\hat{V} e^{i \frac{t+t_{0}}{2} \omega}+\hat{V}^{\dagger} e^{-i \frac{t+t_{0}}{2} \omega}\right) \\
A^{2} & =\left[\hat{V}^{2} e^{i\left(t+t_{0}\right) \omega}+\left(\hat{V}^{\dagger}\right)^{2} e^{-i\left(t+t_{0}\right) \omega}-\left(\left\{V, V^{\dagger}\right\}\right)\right] f^{2}(t, \omega),
\end{aligned}
$$

where $\left\{V, V^{\dagger}\right\}=V V^{\dagger}+V^{\dagger} V$. At this point we are ready to perform the traces. First, we have that

$$
\operatorname{Tr}(A)=-\left[\operatorname{Tr}(\hat{V}) e^{i \frac{t+t_{0}}{2} \omega}+\operatorname{Tr}\left(\hat{V}^{\dagger}\right) e^{-i \frac{t+t_{0}}{2} \omega}\right] f(t, \omega) .
$$

Let $\lambda_{k}$ be the complex eigenvalues of $\hat{V}$ and $\sigma_{k}$ the singular values. Then, we have

$$
\begin{aligned}
\operatorname{Tr}(A) & =-\left[\operatorname{Tr}(\hat{V}) e^{i \frac{t+t_{0}}{2} \omega}+\operatorname{Tr}\left(\hat{V}^{\dagger}\right) e^{-i \frac{t+t_{0}}{2} \omega}\right] f(t, \omega) \\
& =-2 \sum_{k} \operatorname{Re}\left(\lambda_{k} e^{i \frac{t+t_{0}}{2} \omega}\right) f(t, \omega),
\end{aligned}
$$

meanwhile

$$
\operatorname{Tr}\left(A^{2}\right)=-2\left\{\sum_{k} \operatorname{Re}\left[\lambda_{k}^{2} e^{i \omega\left(t_{0}+t\right)}\right]-\sum_{k} \sigma_{k}\right\} f^{2}(t, \omega) .
$$

And thus the $A$-dependent part of the average work is given by

$$
\begin{aligned}
\left\langle\Delta A^{2}\right\rangle_{G}= & \frac{2 f^{2}(t, \omega)}{\left(n^{2}-1\right)}\left\{\sum_{k, k^{\prime}} \operatorname{Re}\left(\lambda_{k} e^{i \frac{t+t_{0}}{2} \omega}\right) \operatorname{Re}\left(\lambda_{k^{\prime}} e^{i \frac{t+t_{0}}{2} \omega}\right)\right. \\
& \left.-2 n \sum_{k} \operatorname{Re}\left[\lambda_{k}^{2} e^{i \omega\left(t_{0}+t\right)}\right]+2 n \sum_{k} \sigma_{k}\right\}, \quad(\mathrm{B}
\end{aligned}
$$

which is the expression for the performed work due to a harmonic perturbation. What we see is that the overall work is proportional to product of two functions, where one is the square of function $f(t, \omega)=2 \frac{\sin \left(\frac{t t_{0}}{2} \omega\right)}{\omega}$ and a factor which depends on the eigenvalues of the operator $\hat{V}$. The function $f$ is periodic with period $\frac{2 \pi}{\omega}$ and has a maximum for $t_{k}=$ $(4 k+1) \frac{\pi}{\omega}+t_{0}$. If $\hat{V}$ is self-adjoint, $\sigma_{k}=\lambda_{k}^{2}$, and we have in the parenthesis the function

$$
\sum_{k, k^{\prime}} \lambda_{k} \lambda_{k^{\prime}} \cos ^{2}\left(\frac{t+t_{0}}{2} \omega\right)+2 n \sum_{k} \lambda_{k}^{2}\left[1-\cos ^{2}\left(\frac{t+t_{0}}{2} \omega\right)\right],
$$

which can be rewritten as

$$
2 n \sum_{k} \lambda_{k}^{2}+\cos ^{2}\left(\frac{t+t_{0}}{2} \omega\right) \sum_{k \neq k^{\prime}} \lambda_{k} \lambda_{k^{\prime}} .
$$

If we introduce the constants $a_{0}, b_{0}, c_{0}$, then the work is thus a function of the form:

$$
a_{0} \sin ^{2}\left(x-x_{0}\right)\left[c_{0}+d_{0} \cos ^{2}\left(x+x_{0}\right)\right],
$$

which is periodic. For $t \gg t_{0}$, the function above has two minima if $c_{0}<d_{0}$ and only one for $c_{0}>d_{0}$. However, it is not hard to see that $c_{0}>d_{0}$ is always true if

$$
\operatorname{Tr}\left(A^{2}\right)-\frac{\operatorname{Tr}(A)^{2}}{2 n+1} \geqslant 0
$$


is always true $\forall A$. However, the identity above follows immediately from the fact that

$$
\operatorname{Tr}(a A+b I)^{2} \geqslant 0
$$

is true for arbitrary $a, b \in \mathbb{R}$, and it follows from the choice $a=n, b=c_{ \pm} \operatorname{Tr}(A)$ with

$$
c_{ \pm}=-1 \pm \sqrt{1-\frac{n}{2 n+1}} .
$$

Thus, the work performed by a (random) harmonic perturbation of the form $2 \hat{V} \cos (\omega t)$ has always a single maximum at $t_{k}=(2 k+1) \frac{\pi}{\omega}$ on average. This can be interpreted as the fact that there are specific moments at which we stop our process to have performed the maximum amount of work on the battery.

\section{Random spacing for CUE ensemble}

Consider the following problem. Given the function

$$
Q=\sum_{i=1}^{n} \sum_{j=i+1}^{n} \cos \left(\theta_{i}-\theta_{j}\right)
$$

with $\lambda_{j}=e^{i \theta_{j}}$, we ask what is the approximate value of $Q$ for a random matrix in the CUE. First, we note that we can write

$$
\begin{aligned}
Q & =\sum_{k=1}^{n} \sum_{j=k+1}^{n} \cos \left(-i \log \lambda_{k}-\log \lambda_{j}\right) \\
& =\sum_{k=1}^{n} \sum_{j=k+1}^{n} \cosh \left[\log \left(\frac{\lambda_{k}}{\lambda_{j}}\right)\right] \\
& =\sum_{k=1}^{n} \sum_{j=k+1}^{n} \cosh \left[\log \left(\frac{\lambda_{k}}{\lambda_{j}}\right)\right]=\frac{1}{2} \sum_{k=1}^{n} \sum_{j=k+1}^{n}\left(\frac{\lambda_{k}}{\lambda_{j}}+\frac{\lambda_{j}}{\lambda_{k}}\right) .
\end{aligned}
$$

Let us define $r_{k}=\frac{\lambda_{k+1}}{\lambda_{k}}$. We then see that we can write

$$
\frac{\lambda_{k+t}}{\lambda_{k}}=\prod_{j=0}^{t-1} r_{k+j}
$$

and thus

$$
Q=\frac{1}{2} \sum_{k=1}^{n} \sum_{j=k+1}^{n}\left(\prod_{i=j+1}^{n} r_{i}+\prod_{i=j+1}^{n} r_{i}^{-1}\right)
$$

the average of $Q$, evaluated numerically, is provided in Fig. 1. We see that for large values of $n$ the peak of the distribution moves toward zero.

\section{Adiabatic quantum batteries}

Here we give the details for the calculation of work fluctuations $\Delta W_{a d}^{2}$ for the adiabatic batteries. We first recall the calculation of the average. Let us start from the following protocol. The Hamiltonian, for $\alpha=0,1$, is written for an adiabatic transformation as

$$
H_{\alpha}=\sum_{i=1}^{R} \epsilon_{\alpha}^{i} \Pi_{\alpha}^{i}
$$

Consider $\epsilon^{i}(t):[0,1] \rightarrow \mathbb{R}$, with $\epsilon^{i}(0)=\epsilon_{0}^{i}, \epsilon^{i}(1)=\epsilon_{1}^{i}$. It can be shown that the evolution of the projector operators can be written as

$$
\Pi_{\alpha}^{i}(t)=U_{t} \Pi_{\alpha}^{i}(0) U_{t}^{\dagger} .
$$

Thus, the time evolution of the Hamiltonian for an adiabatic system can be written as

$$
H(t)=\sum_{i=1}^{R} \epsilon^{i}(t) U_{t} \Pi_{0}^{i} U_{t}^{\dagger},
$$

where the while the density matrix as $\rho(t)=\sum_{i} p_{i} U_{t} \Pi_{0}^{i} U_{t}^{\dagger}$. It is important that the vector $d_{i}^{\alpha} \equiv\left(\operatorname{Tr}\left(\Pi_{\alpha}^{j}\right)\right.$ does not change with time and thus can simply call $d_{i}$ these quantities; meanwhile, $n$ is the dimension of the Hilbert space.

Because these relationships are in a way independent from the intermediate states, we simply write these expressions for $t=0$ and $t=1$ without loss of generality. The work as

$$
\begin{aligned}
W & =\operatorname{Tr}\left(\rho_{0} H_{0}\right)-\operatorname{Tr}\left(\rho_{1} H_{0}\right) \\
& =\sum_{i=1}^{R} \operatorname{Tr}\left[p_{i}\left(\Pi_{0}^{i}-\Pi_{1}^{i}\right) H_{0}\right] \\
& =\sum_{i, j=1}^{R} p_{i} \epsilon_{0}^{j} \operatorname{Tr}\left[\left(\Pi_{0}^{i}-\Pi_{1}^{i}\right) \Pi_{0}^{j}\right] .
\end{aligned}
$$

We now have $\Pi_{\alpha}^{i} \Pi_{\beta}^{j}=\delta^{i j}$ if $\alpha=\beta$, but otherwise they are not necessarily orthogonal. Let us write the work as

$$
\begin{aligned}
W & =\sum_{i, j} p_{i} \epsilon_{0}^{j}\left[\operatorname{Tr}\left(\Pi_{0}^{i} \Pi_{0}^{j}\right)-\operatorname{Tr}\left(\Pi_{0}^{i} \Pi_{1}^{j}\right)\right] \\
& =\sum_{i, j} p_{i} \epsilon_{0}^{j}\left[d_{i} \delta_{i j}-\operatorname{Tr}\left(\Pi_{0}^{i} G \Pi_{0}^{j} G^{\dagger}\right)\right] .
\end{aligned}
$$

We can now perform the average over the unitary transformation $U$. We obtain

$$
\begin{aligned}
\bar{W} & =\sum_{i, j} p_{i} \epsilon_{0}^{j}\left[d_{i} \delta_{i j}-\operatorname{Tr}\left(\Pi_{0}^{i} \frac{d_{j} \mathbb{I}}{n}\right)\right] \\
& =\sum_{i, j} p_{i} \epsilon_{0}^{j}\left(d_{i} \delta_{i j}-\frac{d_{i} d_{j}}{n}\right) .
\end{aligned}
$$

Since we will need it for the calculation of the fluctuations, we note that

$$
\bar{W}^{2}=\sum_{i, j, k, l} p_{i} \epsilon_{0}^{j} p_{k} \epsilon_{0}^{l}\left(d_{i} d_{k} \delta_{i j} \delta_{k l}+\frac{d_{i} d_{j} d_{k} d_{l}}{n^{2}}-\frac{d_{i} d_{j} d_{k} \delta_{k l}+d_{l} d_{k} d_{i} \delta_{i j}}{n}\right) .
$$


Let us now calculate the fluctuations. The square of the work reads

$$
\begin{aligned}
W^{2} & =\sum_{i, j, k, l} p_{i} \epsilon_{0}^{j} p_{k} \epsilon_{0}^{l}\left[d_{i} \delta_{i j}-\operatorname{Tr}\left(\Pi_{0}^{i} G \Pi_{0}^{j} G^{\dagger}\right)\right]\left[d_{k} \delta_{k l}-\operatorname{Tr}\left(\Pi_{0}^{k} G \Pi_{0}^{l} G^{\dagger}\right)\right] \\
& =\sum_{i, j, k, l} p_{i} \epsilon_{0}^{j} p_{k} \epsilon_{0}^{l}\left[d_{i} d_{k} \delta_{i j} \delta_{k l}-d_{i} \delta_{i j} \operatorname{Tr}\left(\Pi_{0}^{k} G \Pi_{0}^{l} G^{\dagger}\right)-d_{k} \delta_{k l} \operatorname{Tr}\left(\Pi_{0}^{i} G \Pi_{0}^{j} G^{\dagger}\right)+\operatorname{Tr}\left(\Pi_{0}^{k} G \Pi_{0}^{l} G^{\dagger}\right) \operatorname{Tr}\left(\Pi_{0}^{i} G \Pi_{0}^{j} G^{\dagger}\right)\right] \\
& =\sum_{i, j, k, l} p_{i} \epsilon_{0}^{j} p_{k} \epsilon_{0}^{l}\left\{d_{i} d_{k} \delta_{i j} \delta_{k l}-d_{i} \delta_{i j} \operatorname{Tr}\left(\Pi_{0}^{k} G \Pi_{0}^{l} G^{\dagger}\right)-d_{k} \delta_{k l} \operatorname{Tr}\left(\Pi_{0}^{i} G \Pi_{0}^{j} G^{\dagger}\right)+\operatorname{Tr}\left[\left(\Pi_{0}^{k} \otimes \Pi_{0}^{i}\right)(G \otimes G)\left(\Pi_{0}^{l} \otimes \Pi_{0}^{j}\right)\left(G^{\dagger} \otimes G^{\dagger}\right)\right]\right\} .
\end{aligned}
$$

We can now perform the averages. We obtain

$$
\begin{aligned}
\left\langle W^{2}\right\rangle_{a d} & =\sum_{i, j, k, l} p_{i} \epsilon_{0}^{j} p_{k} \epsilon_{0}^{l}\left[d_{i} d_{k} \delta_{i j} \delta_{k l}-\left(d_{i} \delta_{i j} \frac{d_{k} d_{l}}{n}+d_{k} \delta_{k l} \frac{d_{i} d_{j}}{n}\right)+\operatorname{Tr}\left[\left(\Pi_{0}^{k} \otimes \Pi_{0}^{i}\right)\left(\lambda_{+} \Pi_{+}+\lambda_{i} \Pi_{-}\right)\right]\right] \\
& =\bar{W}^{2}+\sum_{i, j, k, l} p_{i} \epsilon_{0}^{j} p_{k} \epsilon_{0}^{l}\left(\operatorname{Tr}\left(\Pi_{0}^{k} \otimes \Pi_{0}^{i}\right)\left(\lambda_{+} \Pi_{+}+\lambda_{i} \Pi_{-}\right)-\frac{d_{i} d_{j} d_{k} d_{l}}{n^{2}}\right),
\end{aligned}
$$

where

$$
\lambda_{ \pm}=\frac{\operatorname{Tr}\left(\Pi_{0}^{l} \otimes \Pi_{0}^{j}\right) \Pi_{ \pm}}{\operatorname{Tr} \Pi_{ \pm}}=\frac{d_{l} d_{j} \pm d_{l} \delta_{l j}}{n(n \pm 1)} .
$$

Let us focus on

$$
\operatorname{Tr}\left[\left(\Pi_{0}^{k} \otimes \Pi_{0}^{i}\right)\left(\lambda_{+} \Pi_{+}+\lambda_{i} \Pi_{-}\right)\right]=\operatorname{Tr}\left[\left(\Pi_{0}^{k} \otimes \Pi_{0}^{i}\right)\left(\frac{\lambda_{+}+\lambda_{-}}{2} \mathbb{I}+\frac{\lambda_{+}-\lambda_{-}}{2} \mathbb{T}\right)\right]=\frac{\lambda_{+}+\lambda_{-}}{2} d_{k} d_{i}+\frac{\lambda_{+}-\lambda_{-}}{2} d_{k} \delta_{k i} .
$$

aWe note that

$$
\begin{aligned}
& \frac{\lambda_{+}+\lambda_{-}}{2}=\frac{1}{2}\left[\frac{d_{l} d_{j}+d_{l} \delta_{l j}}{n(n+1)}+\frac{d_{l} d_{j}-d_{l} \delta_{l j}}{n(n-1)}\right]=\frac{d_{l}\left(d d_{j}-\delta_{l j}\right)}{n\left(n^{2}-1\right)} \\
& \frac{\lambda_{+}-\lambda_{-}}{2}=\frac{1}{2} \frac{d_{j} d_{l}+d_{l} \delta_{l j}}{n(n+1)}-\frac{d_{j} d_{l}-d_{l} \delta_{l j}}{(n-1) n}=\frac{d_{l}\left(d \delta_{l j}-d_{j}\right)}{n\left(n^{2}-1\right)}
\end{aligned}
$$

from which we obtain:

$$
\begin{aligned}
\operatorname{Tr}\left[\left(\Pi_{0}^{k} \otimes \Pi_{0}^{i}\right)\left(\lambda_{+} \Pi_{+}+\lambda_{i} \Pi_{-}\right)\right] & =d_{k} d_{l}\left[\frac{d_{i}\left(d d_{j}-\delta_{l j}\right)}{n\left(n^{2}-1\right)}+\frac{\delta_{k i}\left(d \delta_{l j}-d_{j}\right)}{n\left(n^{2}-1\right)}\right] \\
& =\frac{d_{i} d_{j} d_{k} d_{l}}{n^{2}-1}-\frac{d_{i} d_{k} d_{l} \delta_{l j}}{n\left(n^{2}-1\right)}-\frac{d_{j} d_{k} d_{l} \delta_{k i}}{n\left(n^{2}-1\right)}+\frac{d_{k} d_{l} \delta_{k i} \delta_{l j}}{n^{2}-1} .
\end{aligned}
$$

We use the result on $\bar{W}^{2}$, and thus

$$
\overline{W^{2}}-\bar{W}^{2}=\sum_{i, j, k, l} p_{i} \epsilon_{0}^{j} p_{k} \epsilon_{0}^{l}\left[\frac{d_{i} d_{j} d_{k} d_{l}}{n^{2}-1}-\frac{d_{i} d_{k} d_{l} \delta_{l j}}{n\left(n^{2}-1\right)}-\frac{d_{j} d_{k} d_{l} \delta_{k i}}{n\left(n^{2}-1\right)}+\frac{d_{k} d_{l} \delta_{k i} \delta_{l j}}{n^{2}-1}-\frac{d_{i} d_{j} d_{k} d_{l}}{n^{2}}\right] .
$$

For the dimension of the Hilbert space $n \gg 1$, the terms of order $1 / n^{3}$ go to zero faster than $1 / n^{2}$, and we obtain

$$
\overline{W^{2}}-\bar{W}^{2} \underbrace{=}_{n \gg 1} \frac{1}{n^{2}} \sum_{i, j, k, l} p_{i} \epsilon_{0}^{j} p_{k} \epsilon_{0}^{l}\left(d_{k} d_{l} \delta_{k i} \delta_{l j}\right)=\frac{\operatorname{Tr}\left(H_{0} \rho_{0}\right)^{2}}{n^{2}}=\frac{E_{0}^{2}}{n^{2}},
$$

which exhibits concentration.

Let us now look at bounds on the adiabatic work compared to the mean work for arbitrary random evolutions. We consider

$$
\begin{gathered}
\langle W\rangle_{a d}=E_{0}-\sum_{i j} \frac{p_{i} \epsilon_{0}^{j}}{n} d_{i} d_{j}, \\
\langle W\rangle=E_{0}-\frac{\operatorname{Tr} H_{0}}{n}=E_{0}-\sum_{i j} \frac{p_{i} \epsilon_{0}^{j}}{n} .
\end{gathered}
$$

It is easy to see that

$$
\langle W\rangle_{a d}-\langle W\rangle=\operatorname{Tr}(A B),
$$


where $A_{i j}=d_{i} d_{j}-1$ and $B=\frac{p_{i} \epsilon_{j}}{n}$. We now know that for $A$ non-negative and $B$ arbitrary, we have

$$
\operatorname{Tr}(A B) \leqslant \sigma_{\max }(B) \operatorname{Tr}(A)=\sigma_{\max }(B)\left(\sum_{i} d_{i}^{2}-n\right),
$$

where $\sigma_{\max }(B)$ is the spectral norm of the matrix $B$ [63]. The matrix $B=\frac{p_{i} \epsilon_{j}}{n}$ has only two eigenvalues since it is rank one, which are 0 and $\frac{1}{n} \sum_{i} p_{i} \epsilon_{0}^{i}$. Thus the spectral norm is $\sigma_{\max }(B)=\max \left(0, \frac{1}{n} \sum_{i} p_{i} \epsilon_{0}^{i}\right)$. We thus find that the maximum gain that one can has from degeneracy is

$$
\langle W\rangle_{a d}-\langle W\rangle \leqslant \operatorname{Tr}\left(\rho H_{0}\right) \frac{\sum_{i} d_{i}^{2}-n}{n},
$$

from which we obtain

$$
\langle W\rangle_{a d} \leqslant E_{0}(1+c)-\frac{\operatorname{Tr}\left(H_{0}\right)}{n},
$$

with $c=\frac{\sum_{i} d_{i}^{2}-n}{n}$.

[1] F. Campaioli, F. A. Pollock, and S. Vinjanampathy, in Thermodynamics in the Quantum Regime, Fundamental Theories of Physics, Vol. 195, edited by F. Binder, L. Correa, C. Gogolin, J. Anders, and G. Adesso (Springer, Cham, 2018).

[2] R. Alicki and M. Fannes, Phys. Rev. E 87, 042123 (2013).

[3] D. Ferraro, M. Campisi, G. M. Andolina, V. Pellegrini, and M. Polini, Phys. Rev. Lett. 120, 117702 (2018).

[4] G. M. Andolina, D. Farina, A. Mari, V. Pellegrini, V. Giovannetti, and M. Polini, Phys. Rev. B 98, 205423 (2018).

[5] G. M. Andolina, M. Keck, Andrea Mari, M. Campisi, V. Giovannetti, and M. Polini, Phys. Rev. Lett. 122, 047702 (2019).

[6] G. M. Andolina, M. Keck, A. Mari, V. Giovannetti, and M. Polini, Phys. Rev. B 99, 205437 (2019).

[7] S. Julia-Farre, T. Salamon, A. Riera, M. N. Bera, and M. Lewenstein, arXiv:1811.04005.

[8] T. P. Le, J. Levinsen, K. Modi, M. M. Parish, and F. A. Pollock, Phys. Rev. A 97, 022106 (2018).

[9] L. A. Correa, J. P. Palao, G. Adesso, and D. Alonso, Phys. Rev. E 87, 042131 (2013).

[10] G. Watanabe, B. P. Venkatesh, P. Talkner, and A. del Campo, Phys. Rev. Lett. 118, 050601 (2017).

[11] A. Levy, R. Alicki, and R. Kosloff, Phys. Rev. E 85, 061126 (2012).

[12] J. Åberg, Nat. Commun. 4, 1925 (2013).

[13] A. M. Alhambra, L. Masanes, J. Oppenheim, and C. Perry, Phys. Rev. X 6, 041017 (2016).

[14] A. M. Alhambra, G. Styliaris, N. A. Rodriguez-Briones, J. Sikora, and E. Martin-Martinez, Phys. Rev. Lett. 123, 190601 (2019).

[15] R. Alicki and D. Gelbwaser-Klimovsky, New J. Phys. 17, 115012 (2015).

[16] L. Masanes and J. Oppenheim, Nat. Commun. 8, 14538 (2017).

[17] R. Alicki, J. Phys. A 12, L103 (1979); R. Kosloff, J. Chem. Phys. 80, 1625 (1984).

[18] P. Skrzypczyk, A. J. Short, and S. Popescu, Nat. Commun. 5, 4185 (2014).

[19] N. Linden, S. Popescu, and P. Skrzypczyk, Phys. Rev. Lett. 105, 130401 (2010).
[20] J. Anders and M. Esposito, New J. Phys. 19, 010201 (2017).

[21] C. Sparaciari, J. Oppenheim, and T. Fritz, Phys. Rev. A 96, 052112 (2017).

[22] M. Nath Bera, A. Riera, M. Lewenstein, Z. B. Khanian, and A. Winter, Quantum 3, 121 (2019).

[23] F. G. S. L. Brandão, M. Horodecki, J. Oppenheim, Joseph M. Renes, and R. W. Spekkens, Phys. Rev. Lett. 111, 250404 (2013).

[24] R. Demkowicz-Dobrzański, J. Kołodyński, and M. Guţă, Nat. Commun. 3, 1063 (2012).

[25] M. F. Frenzel, D. Jennings, and T. Rudolph, Phys. Rev. E 90, 052136 (2014).

[26] N. Friis and M. Huber, Quantum 2, 61 (2018).

[27] B. Yan, L. Cincio, and W. H. Zurek, Phys. Rev. Lett. 124, 160603 (2020).

[28] J. Goold, M. Huber, A. Riera, L. del Rio, and P. Skrzypczyk, J. Phys. A: Math. Theor. 49, 143001 (2016).

[29] R. Gallego, J. Eisert, and H. Wilming, New J. Phys. 18, 103017 (2016).

[30] S. Ghosh, T. Chanda, and A. Sen (De), Phys. Rev. A 101, 032115 (2020).

[31] D. Rossini, G.-M. Andolina, and M. Polini, Phys. Rev. B 100, 115142 (2019).

[32] A. Chenu, J. Molina-Vilaplana, and A. del Campo, Quantum 3, 127 (2019).

[33] A. Chenu, I. L. Egusquiza, J. Molina-Vilaplana, and A. del Campo, Sci. Rep. 8, 12634 (2018).

[34] J. G. Richens and L. Masanes, Nat. Commun. 7, 13511 (2016).

[35] S. Popescu, A. J. Short, and A. Winter, Nat. Phys. 2, 754 (2006).

[36] A. E. Allahverdyan, R. Balian, and T. M. Nieuwenhuizen, Europhys. Lett. 67, 565 (2004).

[37] J. Kasprzak et al., Nat. Mater. 9, 304 (2010).

[38] T. Niemczyk et al., Nat. Phys. 6, 772 (2010).

[39] K. Zyczkowski, P. Horodecki, A. Sanpera, and M. Lewenstein, Phys. Rev. A 58, 883 (1998).

[40] A. Hamma, S. Santra, and P. Zanardi, Phys. Rev. Lett. 109, 040502 (2012).

[41] A. Hamma, S. Santra, and P. Zanardi, Phys. Rev. A 86, 052324 (2012). 
[42] R. Alicki, R. Horodecki, P. Horodecki, and R. Horodecki, Open Syst. Inform. Dynam. 11, 205 (2004).

[43] J. Åberg, Phys. Rev. Lett. 113, 150402 (2014).

[44] K. Brandner, M. Bauer, and M. T. Schmid, U Seifert, New J. Phys. 17, 065006 (2015).

[45] G. Francica, J. Goold, and F. Plastina, Phys. Rev. E 99, 042105 (2019).

[46] K. Korzekwa, M. Lostaglio, J. Oppenheim, and D. Jennings, New J. Phys. 18, 023045 (2016).

[47] C. L. Latune, I. Sinayskiy, and F. Petruccione, Sci. Rep. 9, 3191 (2019).

[48] M. Lostaglio, K. Korzekwa, D. Jennings, and T. Rudolph, Phys. Rev. X 5, 021001 (2015).

[49] I. Marvian, R. W. Spekkens, and P. Zanardi, Phys. Rev. A 93, 052331 (2016).

[50] A. Streltsov, G. Adesso, and M. B. Plenio, Rev. Mod. Phys. 89, 041003 (2017).

[51] S. Juliá-Farrè, T. Salamon, A. Riera, M. N. Bera, and M. Lewenstein, arXiv: $1811.04005 \mathrm{v} 2$

[52] D. A. Roberts and B. Yoshida, J. High Energ. Phys. 04 (2017) 121.

[53] A. Harrow, L. Kong, Z. Liu, S. Mehraban, and P. Shor, arXiv:1906.02219.

[54] L. Campos Venuti, N. T. Jacobson, S. Santra, and P. Zanardi, Phys. Rev. Lett. 107, 010403 (2011).

[55] E. T. Jaynes and F. W. Cummings, Proc. IEEE. 51, 89 (1963).
[56] F. C. Binder, S. Vinjanampathy, K. Modi, and J. Goold, New J. Phys. 17, 075015 (2015).

[57] F. Campaioli, F. A. Pollock, F. C. Binder, L. Céleri, J. Goold, S. Vinjanampathy, and K. Modi, Phys. Rev. Lett. 118, 150601 (2017).

[58] E. Farhi, J. Goldstone, S. Gutmann, J. Lapan, A. Lundgren, and D. Preda, Science 292, 472 (2001); T. Albash and D. A. Lidar, Rev. Mod. Phys. 90, 015002 (2018).

[59] G. Quiroz, Phys. Rev. A 99, 062306 (2019).

[60] A. Campo, J. Goold, and M. Paternostro, Sci. Rep. 4, 6208 (2015).

[61] A. C. Santos, B. Çakmak, S. Campbell, and N. T. Zinner, Phys. Rev. E 100, 032107 (2019).

[62] A. Hamma and P. Zanardi, Phys. Rev. A 69, 062319 (2004).

[63] S.-D. Wang, T.-S. Kuo, and C.-F. Hsu, IEEE Trans. Autom. Contr. 31, 654 (1986); J. M. Saniuk and I. B. Rhodes, ibid. 32, 739 (1987); T. Mori, ibid. 33, 1088 (1988).

[64] K. Funo, J.-N. Zhang, C. Chatou, K. Kim, M. Ueda, A. del Campo, Phys. Rev. Lett. 118, 100602 (2017).

[65] G. Styliaris, L. Campos Venuti, and P. Zanardi, Phys. Rev. A 97, 032304 (2018).

[66] A. T. Rezakhani, W.-J. Kuo, A. Hamma, D. A. Lidar, and P. Zanardi, Phys. Rev. Lett. 103, 080502 (2009).

[67] L. Mirsky, Mon. F. Math. 79, 303 (1975); also see the notes on spectral methods available at https://www.overleaf.com/read/ wdxknyfmdnww. 